\title{
Saccharide-Dependent Induction of Chiral Helicity in Achiral Synthetic Hydrogen-Bonding Oligomers
}

\author{
Masahiko Inouye, ${ }^{*}$ Minoru Waki, Hajime Abe
}

Faculty of Pharmaceutical Sciences, Toyama Medical and Pharmaceutical University, Toyama 930-0194, Japan

\section{Contents}

Experimental Section

${ }^{1} \mathrm{H}$ NMR spectra for compounds $1,1 \mathrm{~b}-\mathrm{d}, \mathbf{2}, \mathbf{2 b}, \mathbf{2 e}, \mathbf{3}, \mathbf{3 b}, \mathbf{3 e}-\mathrm{g}, 4,4 \mathrm{~h}, 5,5 \mathrm{~h}, \mathbf{6}, \mathbf{6 h}$,

$12,12 \mathrm{~h}, 18,18 \mathrm{~h}, 24$, and $24 \mathrm{~h}$ 


\section{Experimental Section}

2,6-Dibromo-4-butoxypyridine (1a). ${ }^{1} \quad$ A NaH (1.8 g, 45 mmol; commercial 60\% dispersion was washed thoroughly with hexane prior to use) suspension in THF (100 mL) was cooled with an ice bath, and then to the suspension was added slowly 1-butanol $(3.0 \mathrm{~g}, 41 \mathrm{mmol})$. After the evolution of $\mathrm{H}_{2}$ ceased, 2,6-dibromo-4-nitropyridine ${ }^{2}$ was added in one portion at $0{ }^{\circ} \mathrm{C}$. The reaction mixture was stirred for $10 \mathrm{~h}$, allowing to room temperature. The resulted mixture was quenched with sat. $\mathrm{NH}_{4} \mathrm{Cl}$ and extracted with benzene/hexane $(1: 1)$. The extract was washed with brine, dried over $\mathrm{Na}_{2} \mathrm{SO}_{4}$, and evaporated to yield colorless oil 1a (11 g, 89\%). This product was identical to that prepared previously. ${ }^{1}$

4-Butoxy-2,6-diiodopyridine (1b). This compound was prepared by a modification of the published procedure by Suzuki and Inouye et al. ${ }^{3} \quad$ A mixture of $1 \mathbf{a}(10 \mathrm{~g}, 32 \mathrm{mmol}), \mathrm{CuI}(150 \mathrm{~g}, 0.75 \mathrm{~mol})$, and KI (290 g, $1.75 \mathrm{~mol})$ in DMF $(650 \mathrm{~mL})$ was stirred for $24 \mathrm{~h}$ at $140{ }^{\circ} \mathrm{C}$. The resulted brown mixture was concentrated in vacuo, diluted with $\mathrm{CHCl}_{3}$, and filtered. The precipitate was further extracted with $\mathrm{CHCl}_{3}$ by a Soxhlet extractor, and the extract was combined with the filtrate. The combined $\mathrm{CHCl}_{3}$ extract was evaporated and subjected to silica gel column chromatography (eluent; $\mathrm{CH}_{2} \mathrm{Cl}_{2}$ : hexane $=1: 4$ ) to afford $\mathbf{1 b}$ (12 g, 93\%) as yellow oil. IR (neat) 2956, 2871, 1562, $1522 \mathrm{~cm}^{-1} ;{ }^{1} \mathrm{H}$ NMR $\left(\mathrm{CDCl}_{3}, 300 \mathrm{MHz}\right) \delta 0.97(\mathrm{t}, J$ $=7.4 \mathrm{~Hz}, 3 \mathrm{H}), 1.40-1.50(\mathrm{~m}, 2 \mathrm{H}), 1.70-1.79(\mathrm{~m}, 2 \mathrm{H}), 3.96(\mathrm{t}, J=6.5 \mathrm{~Hz}, 2 \mathrm{H}), 7.21(\mathrm{~s}, 2 \mathrm{H}) ;{ }^{13} \mathrm{C} \mathrm{NMR}$ $\left(\mathrm{CDCl}_{3}, 75 \mathrm{MHz}\right) \delta 13.99,19.26,30.91,68.72,115.87,121.27,164.96$; ESI-HRMS $m / z$ calcd for $\mathrm{C}_{9} \mathrm{H}_{12} \mathrm{I}_{2} \mathrm{NO}$ $\left(\mathrm{M}+\mathrm{H}^{+}\right)$: 403.9008; found: 403.9044 .

4-Butoxy-2,6-bis(3-hydroxy-3-methyl-1-butynyl)pyridine (1c). To an $\mathrm{Et}_{2} \mathrm{NH}(100 \mathrm{~mL})$ suspension of $\mathrm{PdCl}_{2}\left(\mathrm{PPh}_{3}\right)_{2}(454 \mathrm{mg}, 0.65 \mathrm{mmol})$ and $\mathrm{CuI}(61 \mathrm{mg}, 0.32 \mathrm{mmol})$ were added $1 \mathrm{a}(5.0 \mathrm{~g}, 16 \mathrm{mmol})$ and 2-methyl-3-butyn-2-ol (3.0 g, $36 \mathrm{mmol})$ subsequently. The mixture was stirred for $3.5 \mathrm{~h}$ at room temperature and evaporated. The evaporated residue was diluted with ether and filtered off the insoluble salts. The filtrate was concentrated and subjected to silica gel column chromatography (eluent; AcOEt : $\left.\mathrm{CH}_{2} \mathrm{Cl}_{2}=1: 1\right)$ to afford $1 \mathrm{c}(5.1 \mathrm{~g}, 100 \%)$ as brownish oil. IR (nujol) $1510 \mathrm{~cm}^{-1} ;{ }^{1} \mathrm{H} \mathrm{NMR}\left(\mathrm{CDCl}_{3}, 300\right.$ MHz) $\delta 0.97(\mathrm{t}, J=7.4 \mathrm{~Hz}, 3 \mathrm{H}), 1.42-1.52(\mathrm{~m}, 2 \mathrm{H}), 1.61(\mathrm{~s}, 12 \mathrm{H}), 1.70-1.82(\mathrm{~m}, 2 \mathrm{H}), 3.98(\mathrm{t}, J=6.5 \mathrm{~Hz}$, 
$2 \mathrm{H}), 4.2$ (br s, $2 \mathrm{H}), 6.84(\mathrm{~s}, 2 \mathrm{H}) ;{ }^{13} \mathrm{C} \mathrm{NMR}\left(\mathrm{CDCl}_{3}, 75 \mathrm{MHz}\right) \delta 13.99,19.31,30.99,31.32,65.13,68.33$, 80.94, 94.69, 113.12, 144.04, 165.35; ESI-HRMS $m / z$ calcd for $\mathrm{C}_{19} \mathrm{H}_{26} \mathrm{NO}_{3}\left(\mathrm{M}+\mathrm{H}^{+}\right)$: 316.1913; found: 316.1888 .

4-Butoxy-2,6-diethynylpyridine (1d). To a $\mathrm{NaH}(64 \mathrm{mg}, 1.6 \mathrm{mmol}$; commercial 60\% dispersion was washed thoroughly with hexane prior to use) suspension in toluene $(200 \mathrm{~mL})$ was added $1 \mathrm{c}(5.1 \mathrm{~g}, 16 \mathrm{mmol})$. The mixture was refluxed for $1 \mathrm{~h}$ and evaporated. The evaporated residue was subjected to silica gel column chromatography (eluent; $\left.\mathrm{CH}_{2} \mathrm{Cl}_{2}\right)$ to afford $\mathbf{1 d}(2.7 \mathrm{~g}, 83 \%)$ as brownish solid. $\mathrm{Mp} 98-100{ }^{\circ} \mathrm{C}$; IR (neat) 3280, 3167, 2960, 2877, 2105, 1585, $1552 \mathrm{~cm}^{-1} ;{ }^{1} \mathrm{H}$ NMR $\left(\mathrm{CDCl}_{3}, 300 \mathrm{MHz}\right) \delta 0.98(\mathrm{t}, J=7.4 \mathrm{~Hz}, 3$ $\mathrm{H}), 1.42-1.54(\mathrm{~m}, 2 \mathrm{H}), 1.72-1.83(\mathrm{~m}, 2 \mathrm{H}), 3.10(\mathrm{~s}, 2 \mathrm{H}), 4.01(\mathrm{t}, J=6.6 \mathrm{~Hz}, 2 \mathrm{H}), 6.97(\mathrm{~s}, 2 \mathrm{H}) ;{ }^{13} \mathrm{C}$ NMR $\left(\mathrm{CDCl}_{3}, 75 \mathrm{MHz}\right) \delta 14.00,19.34,31.02,68.45,77.27,82.43,114.19,143.73,165.35 ;$ ESI-HRMS $\mathrm{m} / z$ calcd for $\mathrm{C}_{26} \mathrm{H}_{26} \mathrm{~N}_{2} \mathrm{NaO}_{2}\left(2 \mathrm{M}+\mathrm{Na}^{+}\right)$: 421.1892; found: 421.1962 .

4-Butoxy-2,6-bis[(4-butoxy-6-iodo-2-pyridyl)ethynyl]pyridine $\quad(\mathbf{3 b}) . \quad$ To an $i-\mathrm{Pr}_{2} \mathrm{NH} \quad\left(\begin{array}{lll}120 & \mathrm{~mL}\end{array}\right)$ suspension of $\mathrm{Pd}_{2}(\mathrm{dba})_{3} \cdot \mathrm{CHCl}_{3}(128 \mathrm{mg}, 0.12 \mathrm{mmol}), \mathrm{PPh}_{3}(129 \mathrm{mg}, 0.49 \mathrm{mmol})$, and $\mathrm{CuI}(23 \mathrm{mg}, 0.123$ mmol $)$ were added $\mathbf{1 b}(8.3 \mathrm{~g}, 21 \mathrm{mmol})$ and $\mathbf{1 c}(0.8 \mathrm{~g}, 4.1 \mathrm{mmol})$ subsequently. The mixture was stirred for $3 \mathrm{~h}$ at room temperature, diluted with $\mathrm{Et}_{2} \mathrm{O}$, and filtered off the insoluble salts. The filtrate was evaporated and subjected to silica gel column chromatography (eluent; $\mathrm{CH}_{2} \mathrm{Cl}_{2}:$ hexane $\left.=1: 4\right)$ to recover $\mathbf{1 b}(4.7 \mathrm{~g}$, $66 \%$ recovery) and $\left(\mathrm{CH}_{2} \mathrm{Cl}_{2}:\right.$ hexane $\left.=1: 1\right)$ to afford $\mathbf{3 b}(2.5 \mathrm{~g}, 82 \%$ based on $\mathbf{1 c})$ as yellow oil. IR (nujol) 1590, 1550, $1195 \mathrm{~cm}^{-1} ;{ }^{1} \mathrm{H}$ NMR $\left(\mathrm{CDCl}_{3}, 300 \mathrm{MHz}\right) \delta 0.94-1.01(\mathrm{~m}, 9 \mathrm{H}), 1.42-1.52(\mathrm{~m}, 6 \mathrm{H}), 1.72-1.82$ (m, $6 \mathrm{H}), 3.96-4.06(\mathrm{~m}, 6 \mathrm{H}), 7.12(\mathrm{~d}, J=2.4 \mathrm{~Hz}, 2 \mathrm{H}), 7.13(\mathrm{~s}, 2 \mathrm{H}), 7.24(\mathrm{~d}, J=2.4 \mathrm{~Hz}, 2 \mathrm{H}) ;{ }^{13} \mathrm{C}$ NMR $\left(\mathrm{CDCl}_{3}, 75 \mathrm{MHz}\right) \delta 13.86,19.15,19.18,30.78,68.56,86.71,88.04,114.49,114.58,117.70,121.39,143.37$, 143.57, 164.78, 165.17; ESI-HRMS $m / z$ calcd for $\mathrm{C}_{31} \mathrm{H}_{34} \mathrm{I}_{2} \mathrm{~N}_{3} \mathrm{O}_{3}\left(\mathrm{M}+\mathrm{H}^{+}\right):$750.0690; found: 750.0714 .

2-(2-Hydroxypropyl)-protected $\boldsymbol{m}$-Ethynylpyridine Trimer 3e. To an $i$ - $\mathrm{Pr}_{2} \mathrm{NH}(45 \mathrm{~mL})$ suspension of $\mathrm{PdCl}_{2}\left(\mathrm{PPh}_{3}\right)_{2}(116 \mathrm{mg}, 0.17 \mathrm{mmol})$ and $\mathrm{CuI}(16 \mathrm{mg}, 0.083 \mathrm{mmol})$ were added $\mathbf{3 b}(3.1 \mathrm{~g}, 4.1 \mathrm{mmol})$ and 2-methyl-3-butyn-2-ol $(87 \mathrm{mg}, 1.0 \mathrm{mmol})$ subsequently. The mixture was stirred for $3.5 \mathrm{~h}$ at room temperature and concentrated. The concentrated residue was diluted with $\mathrm{Et}_{2} \mathrm{O}$ and filtered off the insoluble salts. The filtrate was evaporated and subjected to silica gel column chromatography (eluent; $\mathrm{CH}_{2} \mathrm{Cl}_{2}$ : 
hexane $=1: 4)$ to recover $\mathbf{3 b}(2.3 \mathrm{~g}, 75 \%$ recovery $)$ and $\left(\mathrm{CH}_{2} \mathrm{Cl}_{2}: \mathrm{MeOH}=100: 1\right)$ to afford $\mathbf{3 e}(0.59 \mathrm{~g}$, $80 \%$ based on 2-methyl-3-butyn-2-ol) as yellow oil. IR (nujol) $1590,1545,1210 \mathrm{~cm}^{-1} ;{ }^{1} \mathrm{H} \mathrm{NMR}\left(\mathrm{CDCl}_{3}\right.$, $300 \mathrm{MHz}) \delta 0.95-1.02(\mathrm{~m}, 9 \mathrm{H}), 1.42-1.52(\mathrm{~m}, 6 \mathrm{H}), 1.53(\mathrm{~s}, 6 \mathrm{H}), 1.73-1.83(\mathrm{~m}, 6 \mathrm{H}), 3.97-4.05(\mathrm{~m}, 6 \mathrm{H})$, $6.92(\mathrm{~d}, J=2.4 \mathrm{~Hz}, 1 \mathrm{H}), 7.09(\mathrm{~d}, J=2.7 \mathrm{~Hz}, 1 \mathrm{H}), 7.12-7.14(\mathrm{~m}, 6 \mathrm{H}), 7.24(\mathrm{~d}, J=2.1 \mathrm{~Hz}, 1 \mathrm{H}) ;{ }^{13} \mathrm{C}$ NMR $\left(\mathrm{CDCl}_{3}, 75 \mathrm{MHz}\right) \delta 13.95,19.25,19.28,30.89,31.28,31.35,65.42,65.52,68.45,68.64,81.20,84.24,86.71$, $87.35,88.25,94.21,113.91,114.06,114.61,114.65,114.72,117.73,121.53,143.57,143.67,143.91,144.38$, 164.94, 165.25, 165.31; ESI-HRMS $m / z$ calcd for $\mathrm{C}_{36} \mathrm{H}_{40} \mathrm{IN}_{3} \mathrm{NaO}_{4}\left(\mathrm{M}+\mathrm{Na}^{+}\right)$: 728.1961; found: 728.1861 .

\section{2-(2-Hydroxypropyl)- and TBDMS-protected $\boldsymbol{m}$-Ethynylpyridine Trimer 3f. $\quad$ To an $i$ - $\operatorname{Pr}_{2} \mathrm{NH}(20 \mathrm{~mL})$} suspension of $\mathrm{PdCl}_{2}\left(\mathrm{PPh}_{3}\right)_{2}(45 \mathrm{mg}, 0.065 \mathrm{mmol})$ and $\mathrm{CuI}(6 \mathrm{mg}, 0.032 \mathrm{mmol})$ were added $3 \mathrm{e}(1.1 \mathrm{~g}, 1.6$ mmol) and (tert-butyldimethylsilyl)acetylene $(1.1 \mathrm{~g}, 8.0 \mathrm{mmol})$ subsequently. The mixture was stirred for 3 $\mathrm{h}$ at room temperature and concentrated. The concentrated residue was diluted with $\mathrm{Et}_{2} \mathrm{O}$ and filtered off the insoluble salts. The filtrate was evaporated and subjected to silica gel column chromatography (eluent; $\mathrm{CH}_{2} \mathrm{Cl}_{2}$ : hexane $=1: 1$ to $\mathrm{CH}_{2} \mathrm{Cl}_{2}$ only) to afford $\mathbf{3 f}(1.1 \mathrm{~g}, 91 \%)$ as yellow solid. $\mathrm{Mp} 54-56{ }^{\circ} \mathrm{C}$; IR (nujol) $1535 \mathrm{~cm}^{-1} ;{ }^{1} \mathrm{H}$ NMR $\left(\mathrm{CDCl}_{3}, 300 \mathrm{MHz}\right) \delta 0.20(\mathrm{~s}, 6 \mathrm{H}), 0.95-1.04(\mathrm{~m}, 9 \mathrm{H}), 1.01(\mathrm{~s}, 9 \mathrm{H}), 1.44-1.53(\mathrm{~m}, 6$ H), $1.53(\mathrm{~s}, 6 \mathrm{H}), 1.74-1.84(\mathrm{~m}, 6 \mathrm{H}), 3.98-4.06(\mathrm{~m}, 6 \mathrm{H}), 6.92(\mathrm{~d}, J=2.1 \mathrm{~Hz}, 1 \mathrm{H}), 6.97(\mathrm{~d}, J=2.7 \mathrm{~Hz}, 1 \mathrm{H})$, $7.10(\mathrm{~d}, J=2.1 \mathrm{~Hz}, 1 \mathrm{H}), 7.12(\mathrm{~d}, J=2.7 \mathrm{~Hz}, 1 \mathrm{H}), 7.14(\mathrm{~s}, 2 \mathrm{H}) ;{ }^{13} \mathrm{C} \mathrm{NMR}\left(\mathrm{CDCl}_{3}, 75 \mathrm{MHz}\right) \delta-4.52,13.92$, $16.88,19.25,26.35,30.88,30.91,31.27,31.33,65.39,65.47,68.38,68.43,68.58,81.16,84.21,87.25,87.43$, $87.52,87.72,94.22,113.85,114.03,114.59,114.66,114.77,143.59,143.73,143.80,143.88,144.33,144.44$, 165.14, 165.22, 165.28; ESI-HRMS $m / z$ calcd for $\mathrm{C}_{44} \mathrm{H}_{56} \mathrm{~N}_{3} \mathrm{O}_{4} \mathrm{Si}\left(\mathrm{M}+\mathrm{H}^{+}\right)$: 718.4040; found: 718.4033 .

TBDMS-protected $\boldsymbol{m}$-Ethynylpyridine Trimer $3 g$. To a $\mathrm{NaH}(110 \mathrm{mg}, 2.7 \mathrm{mmol}$; commercial $60 \%$ dispersion was washed thoroughly with hexane prior to use) suspension in toluene ( $80 \mathrm{~mL})$ was added $\mathbf{3 f}$ (965 mg, $1.34 \mathrm{mmol}$ ). The mixture was refluxed for $30 \mathrm{~min}$ and evaporated. The evaporated residue was subjected to silica gel column chromatography (eluent; $\mathrm{CH}_{2} \mathrm{Cl}_{2}$ : hexane $\left.=1: 1\right)$ to afford $\mathbf{3 g}(0.7 \mathrm{~g}, 80 \%)$ as brown solid. $\operatorname{Mp~36-39~}{ }^{\circ} \mathrm{C}$; IR (nujol) 1590, 1545, $1220 \mathrm{~cm}^{-1} ;{ }^{1} \mathrm{H} \mathrm{NMR}\left(\mathrm{CDCl}_{3}, 300 \mathrm{MHz}\right) \delta 0.20(\mathrm{~s}, 6 \mathrm{H})$, 0.94-1.03 (m, $18 \mathrm{H}), 1.43-1.53(\mathrm{~m}, 6 \mathrm{H}), 1.74-1.83(\mathrm{~m}, 6 \mathrm{H}), 3.13(\mathrm{~s}, 1 \mathrm{H}), 3.99-4.05(\mathrm{~m}, 6 \mathrm{H}), 6.97$ (d, $J=$ $2.1 \mathrm{~Hz}, 1 \mathrm{H}), 6.99$ (d, $J=2.1 \mathrm{~Hz}, 1 \mathrm{H}), 7.10(\mathrm{~d}, J=2.1 \mathrm{~Hz}, 1 \mathrm{H}), 7.13-7.14(\mathrm{~m}, 3 \mathrm{H}) ;{ }^{13} \mathrm{C} \mathrm{NMR}\left(\mathrm{CDCl}_{3}, 75\right.$ 
MHz) $\delta-4.47,13.97,16.93,19.31,26.40,30.93,30.98,68.40,68.50,68.59,77.31,82.38,87.28,87.39$, $87.67,113.85,114.09,114.67,114.79,143.65,143.80,143.88,143.97,144.52,165.17,165.31$; ESI-HRMS $m / z$ calcd for $\mathrm{C}_{41} \mathrm{H}_{49} \mathrm{~N}_{3} \mathrm{NaO}_{3} \mathrm{Si}\left(\mathrm{M}+\mathrm{Na}^{+}\right)$: 682.3441; found: 682.3357 .

TBDMS-protected $\boldsymbol{m}$-Ethynylpyridine Hexamer $6 \mathbf{h}$. To an $i-\mathrm{Pr}_{2} \mathrm{NH}(65 \mathrm{~mL})$ and $\mathrm{DMF}(15 \mathrm{~mL})$ suspension of $\mathrm{Pd}_{2}(\mathrm{dba})_{3} \cdot \mathrm{CHCl}_{3}(55 \mathrm{mg}, 0.053 \mathrm{mmol}), \mathrm{PPh}_{3}(56 \mathrm{mg}, 0.21 \mathrm{mmol})$, and $\mathrm{CuI}(5.1 \mathrm{mg}, 0.027$ mmol) were added $\mathbf{3 b}(4.0 \mathrm{~g}, 5.3 \mathrm{mmol})$ and $\mathbf{3 g}(0.70 \mathrm{~g}, 1.1 \mathrm{mmol})$ subsequently. The mixture was stirred for $2 \mathrm{~h}$ at room temperature and concentrated. The concentrated residue was diluted with $\mathrm{Et}_{2} \mathrm{O}$ and filtered off the insoluble salts. The filtrate was evaporated and subjected to silica gel column chromatography (eluent; $\mathrm{CH}_{2} \mathrm{Cl}_{2}:$ hexane $\left.=1: 4\right)$ to recover $3 \mathbf{b}(3.0 \mathrm{~g}, 92 \%$ recovery $)$ and $\left(\mathrm{CH}_{2} \mathrm{Cl}_{2}:\right.$ hexane $\left.=1: 1\right)$ to afford

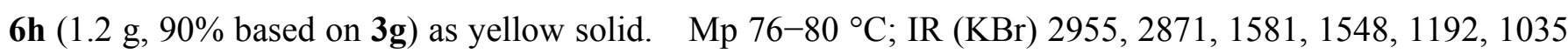
$\mathrm{cm}^{-1} ;{ }^{1} \mathrm{H}$ NMR $\left(\mathrm{CDCl}_{3}, 300 \mathrm{MHz}\right) \delta 0.20(\mathrm{~s}, 6 \mathrm{H}), 0.96-1.04(\mathrm{~m}, 27 \mathrm{H}), 1.44-1.54(\mathrm{~m}, 12 \mathrm{H}), 1.74-1.84(\mathrm{~m}$, $12 \mathrm{H}), 3.97-4.06(\mathrm{~m}, 12 \mathrm{H}), 6.97(\mathrm{~d}, J=2.7 \mathrm{~Hz}, 1 \mathrm{H}), 7.11-7.18(\mathrm{~m}, 10 \mathrm{H}), 7.24(\mathrm{~d}, J=2.1 \mathrm{~Hz}, 1 \mathrm{H}) ;{ }^{13} \mathrm{C}$ NMR $\left(\mathrm{CDCl}_{3}, 75 \mathrm{MHz}\right) \delta-4.46,14.00,16.95,19.33,26.41,30.94,68.43,68.64,87.44,93.56,113.90$, 114.72, 143.95, 143.96, 165.35; ESI-HRMS $m / z$ calcd for $\mathrm{C}_{72} \mathrm{H}_{82} \mathrm{IN}_{6} \mathrm{O}_{6} \mathrm{Si}\left(\mathrm{M}+\mathrm{H}^{+}\right)$: 1281.5110; found: 1281.5069.

m-Ethynylpyridine Hexamer 6. To a THF $(2 \mathrm{~mL})$ solution of $6 \mathbf{h}(0.20 \mathrm{~g}, 0.16 \mathrm{mmol})$ were added $n$ - $\mathrm{Bu}_{4} \mathrm{NF}(1.0 \mathrm{M}$ THF solution, $1.6 \mathrm{~mL}, 1.6 \mathrm{mmol})$ and a few drops of $\mathrm{H}_{2} \mathrm{O}$. The mixture was stirred for 1.5 $\mathrm{h}$ at room temperature, concentrated, and diluted with $\mathrm{CHCl}_{3} / \mathrm{H}_{2} \mathrm{O}$. The separated $\mathrm{CHCl}_{3}$ layer was washed with $\mathrm{H}_{2} \mathrm{O}$, dried over $\mathrm{K}_{2} \mathrm{CO}_{3}$, evaporated, and subjected to silica gel column chromatography (eluent; $\left.\mathrm{CH}_{2} \mathrm{Cl}_{2}: \mathrm{MeOH}=100: 1\right)$ to afford $6(0.16 \mathrm{~g}, 90 \%)$ as yellow solid. Mp $179-180{ }^{\circ} \mathrm{C}$; $\mathrm{IR}(\mathrm{KBr}) 2958,2872$, 1582, 1548, 1191, $1037 \mathrm{~cm}^{-1} ;{ }^{1} \mathrm{H}$ NMR $\left(\mathrm{CDCl}_{3}, 300 \mathrm{MHz}\right) \delta 0.96-1.02(\mathrm{~m}, 18 \mathrm{H}), 1.44-1.54(\mathrm{~m}, 12 \mathrm{H})$, 1.74-1.85 (m, $12 \mathrm{H}), 3.13(\mathrm{~s}, 1 \mathrm{H}), 3.97-4.06(\mathrm{~m}, 12 \mathrm{H}), 7.00(\mathrm{~d}, J=2.4 \mathrm{~Hz}, 1 \mathrm{H}), 7.11-7.18(\mathrm{~m}, 10 \mathrm{H}), 7.24$ $(\mathrm{d}, J=2.4 \mathrm{~Hz}, 1 \mathrm{H}) ;{ }^{13} \mathrm{C} \mathrm{NMR}\left(\mathrm{CDCl}_{3}, 75 \mathrm{MHz}\right) \delta 14.02,19.36,30.99,68.67,87.52,100.77,114.75,144.02$, 165.40; ESI-HRMS $m / z$ calcd for $\mathrm{C}_{66} \mathrm{H}_{68} \mathrm{IN}_{6} \mathrm{O}_{6}\left(\mathrm{M}+\mathrm{H}^{+}\right)$: 1167.4245 ; found: 1167.4069 .

$\boldsymbol{m}$-Ethynylpyridine Polymer $\mathbf{1}_{\text {poly. }} \quad$ To an $i$ - $\operatorname{Pr}_{2} \mathrm{NH}(100 \mathrm{~mL})$ and DMF $(10 \mathrm{~mL})$ suspension of $\mathrm{Pd}_{2}(\mathrm{dba})_{3} \cdot \mathrm{CHCl}_{3}(4.4 \mathrm{mg}, 4.3 \mu \mathrm{mol}), \mathrm{PPh}_{3}(4.5 \mathrm{mg}, 17 \mu \mathrm{mol})$, and $\mathrm{CuI}(0.4 \mathrm{mg}, 2.2 \mu \mathrm{mol})$ was added $6(50$ 
$\mathrm{mg}, 43 \mu \mathrm{mol})$. The mixture was stirred for 4 days at room temperature and filtrated. The precipitate was washed with $\mathrm{Et}_{2} \mathrm{O}, \mathrm{MeOH}$, and $\mathrm{H}_{2} \mathrm{O}$ subsequently, and treated with a Sephadex LH-20 column (eluent; $\mathrm{CHCl}_{3}$ ) to remove tarry impurities. The eluent was evaporated, and the final purification was done by preparative gel permeation chromatography to remove remained $\mathbf{6}$ and dried in vacuo to afford $\mathbf{1}_{\text {poly }}(18 \mathrm{mg}$, $35 \%$ by weight) as brown solid. IR ( $\mathrm{KBr}) 2957,2870,1582,1547,1189,1037 \mathrm{~cm}^{-1} ;{ }^{1} \mathrm{H} \mathrm{NMR}\left(\mathrm{CDCl}_{3}, 300\right.$ MHz) $\delta 1.00(\mathrm{t}, J=7.4 \mathrm{~Hz}, 3 \mathrm{n} \mathrm{H}), 1.44-1.58(\mathrm{~m}, 2 \mathrm{n} \mathrm{H}), 1.76-1.85(\mathrm{~m}, 2 \mathrm{n} \mathrm{H}), 4.04(\mathrm{t}, J=6.5 \mathrm{~Hz}, 2 \mathrm{n} \mathrm{H})$, 7.00-7.30 (m, 2n H); ${ }^{13} \mathrm{C} \mathrm{NMR}\left(\mathrm{CDCl}_{3}, 75 \mathrm{MHz}\right) \delta 13.70,19.04,30.68,68.36,87.22,114.45,143.71$, 165.08 .

4-Butoxy-2-ethynyl-6-iodopyridine (1). To an $\mathrm{Et}_{3} \mathrm{~N}(15 \mathrm{~mL})$ suspension of $\mathrm{PdCl}_{2}\left(\mathrm{PPh}_{3}\right)_{2}(51 \mathrm{mg}, 0.22$ $\mathrm{mmol})$ and $\mathrm{CuI}(14 \mathrm{mg}, 0.22 \mathrm{mmol})$ were added $\mathbf{1 b}(1.7 \mathrm{~g}, 4.3 \mathrm{mmol})$ and (trimethylsilyl)acetylene $(0.14 \mathrm{~g}$, $1.5 \mathrm{mmol}$ ) subsequently. The mixture was stirred for $4 \mathrm{~h}$ at room temperature and concentrated. The concentrated residue was diluted with $\mathrm{Et}_{2} \mathrm{O}$, then filtered through a florisil bed. The filtrate was evaporated and diluted with THF $(10 \mathrm{~mL})$, and then to the filtrate were added $n$-Bu ${ }_{4} \mathrm{NF}$ (1.0 M THF solution, $2.2 \mathrm{~mL}$, $2.2 \mathrm{mmol}$ ) and a few drops of $\mathrm{H}_{2} \mathrm{O}$. The mixture was stirred for $1 \mathrm{~h}$ at room temperature, and then to the mixture was added sat. $\mathrm{NaHCO}_{3}$. The resulted mixture was extracted with AcOEt, and the extract was washed with sat. $\mathrm{NaHCO}_{3}$ and dried over $\mathrm{Na}_{2} \mathrm{SO}_{4}$. The evaporated residue was subjected to silica gel column chromatography (eluent; $\mathrm{CH}_{2} \mathrm{Cl}_{2}$ : hexane = $\left.1: 3\right)$ to afford recovered $\mathbf{1 b}$ followed by $\mathbf{1}(0.20 \mathrm{~g}, 46 \%$ by 2 steps based on (trimethylsilyl)acetylene) as colorless solid. Mp $71-73{ }^{\circ} \mathrm{C}$. IR (nujol) $1545 \mathrm{~cm}^{-1} ;{ }^{1} \mathrm{H}$ NMR $\left(\mathrm{CDCl}_{3}, 300 \mathrm{MHz}\right) \delta 0.97(\mathrm{t}, J=7.4 \mathrm{~Hz}, 3 \mathrm{H}), 1.41-1.52(\mathrm{~m}, 2 \mathrm{H}), 1.69-1.81(\mathrm{~m}, 2 \mathrm{H}), 3.15(\mathrm{~s}, 1 \mathrm{H})$, $3.98(\mathrm{t}, J=6.6 \mathrm{~Hz}, 2 \mathrm{H}), 6.96(\mathrm{~d}, J=2.1 \mathrm{~Hz}, 1 \mathrm{H}), 7.22(\mathrm{~d}, J=2.1 \mathrm{~Hz}, 1 \mathrm{H}) ;{ }^{13} \mathrm{C} \mathrm{NMR}\left(\mathrm{CDCl}_{3}, 75 \mathrm{MHz}\right) \delta$ $13.99,19.29,30.94,68.59,78.24,114.64,117.75,121.13,143.31,164.97$; ESI-HRMS $\mathrm{m} / \mathrm{z}$ calcd for $\mathrm{C}_{11} \mathrm{H}_{12} \mathrm{INNaO}\left(\mathrm{M}+\mathrm{Na}^{+}\right): 323.9861$; found: 323.9915 .

2,2'-(1,2-Ethynediyl)bis(4-butoxy-6-iodopyridine) (2b). A mixture of $\mathbf{1 b}(3.0 \mathrm{~g}, 7.4 \mathrm{mmol})$, bis(tri-n-butylstannyl)acetylene (2.3 g, $3.7 \mathrm{mmol}), \mathrm{Pd}(\mathrm{dba})_{2}(43 \mathrm{mg}, 0.074 \mathrm{mmol})$, and $\mathrm{PPh}_{3}(39 \mathrm{mg}, 0.15$ $\mathrm{mmol})$ in toluene $(80 \mathrm{~mL})$ was stirred for $6 \mathrm{~h}$ at $70{ }^{\circ} \mathrm{C}$. The resulted mixture was concentrated and extracted with $\mathrm{Et}_{2} \mathrm{O}$. The $\mathrm{Et}_{2} \mathrm{O}$ extract was washed with $\mathrm{H}_{2} \mathrm{O}$, dried over $\mathrm{K}_{2} \mathrm{CO}_{3}$, evaporated, and subjected to silica 
gel column chromatography (eluent; $\mathrm{CH}_{2} \mathrm{Cl}_{2}$ : hexane $=1: 4$ to $\mathrm{CH}_{2} \mathrm{Cl}_{2}$ only) to afford $\mathbf{2 b}(0.6 \mathrm{~g}, 28 \%$ based on bis(tri- $n$-butylstannyl)acetylene) as yellow solid. Mp 95-97 ${ }^{\circ} \mathrm{C}$; IR (neat) $1535,1219 \mathrm{~cm}^{-1}$; ${ }^{1} \mathrm{H}$ NMR $\left(\mathrm{CDCl}_{3}, 300 \mathrm{MHz}\right) \delta 0.98(\mathrm{t}, J=7.4 \mathrm{~Hz}, 6 \mathrm{H}), 1.41-1.52(\mathrm{~m}, 4 \mathrm{H}), 1.72-1.82(\mathrm{~m}, 4 \mathrm{H}), 3.99(\mathrm{t}, J=6.5 \mathrm{~Hz}, 4$ $\mathrm{H}), 7.11(\mathrm{~d}, J=2.4 \mathrm{~Hz}, 2 \mathrm{H}), 7.24(\mathrm{~d}, J=2.4 \mathrm{~Hz}, 2 \mathrm{H}) ;{ }^{13} \mathrm{C} \mathrm{NMR}\left(\mathrm{CDCl}_{3}, 75 \mathrm{MHz}\right) \delta 14.00,19.31,30.94$, 68.74, 87.54, 114.64, 117.81, 121.68, 143.50, 165.01; ESI-HRMS $m / z$ calcd for $\mathrm{C}_{20} \mathrm{H}_{22} \mathrm{I}_{2} \mathrm{~N}_{2} \mathrm{NaO}_{2}\left(\mathrm{M}+\mathrm{Na}^{+}\right)$: 598.9669; found: 598.9607.

2-(3-Hydroxy-3-methyl-1-butynyl)-6-(6-iodo-2-pyridylethynyl)pyridine (2e). $\quad$ To an $i$ - $\operatorname{Pr}_{2} \mathrm{NH}(40 \mathrm{~mL})$ suspension of $\mathrm{PdCl}_{2}\left(\mathrm{PPh}_{3}\right)_{2}(83 \mathrm{mg}, 0.12 \mathrm{mmol})$ and $\mathrm{CuI}(11 \mathrm{mg}, 0.059 \mathrm{mmol})$ were added $2 \mathbf{b}(1.7 \mathrm{~g}, 2.9$ mmol) and 2-methyl-3-butyn-2-ol (0.12 g, $1.5 \mathrm{mmol})$ subsequently. The mixture was stirred for $12 \mathrm{~h}$ at room temperature and concentrated. The concentrated residue was diluted with $\mathrm{Et}_{2} \mathrm{O}$ and filtered off the insoluble salts. The filtrate was evaporated and subjected to silica gel column chromatography (eluent; $\mathrm{CH}_{2} \mathrm{Cl}_{2}:$ hexane $\left.=1: 1\right)$ to recover $\mathbf{2 b}$ and $\left(\mathrm{CH}_{2} \mathrm{Cl}_{2}: \mathrm{MeOH}=100: 1\right)$ to afford $\mathbf{2 e}(0.71 \mathrm{~g}, 46 \%$ based on 2-methyl-3-butyn-2-ol) as yellow oil. $\quad$ IR (nujol) $1510 \mathrm{~cm}^{-1} ;{ }^{1} \mathrm{H} \mathrm{NMR}\left(\mathrm{CDCl}_{3}, 300 \mathrm{MHz}\right) \delta 0.99$ (t, $J=7.5$ Hz, 6 H), 1.43-1.54 (m, 4 H), 1.63 (s, 6 H), 1.72-1.83 (m, 4 H), 2.23 (s, 1 H), 3.96-4.03 (m, 4 H), 6.93 (d, J $=2.1 \mathrm{~Hz}, 1 \mathrm{H}), 7.08-7.10(\mathrm{~m}, 2 \mathrm{H}), 7.23(\mathrm{~d}, J=2.1 \mathrm{~Hz}, 1 \mathrm{H}) ;{ }^{13} \mathrm{C} \mathrm{NMR}\left(\mathrm{CDCl}_{3}, 75 \mathrm{MHz}\right) \delta 13.99,19.31$, $19.34,30.94,30.99,31.40,65.65,68.53,68.71,88.40,93.83,113.96,114.30,114.61,117.78,121.58,143.60$, 143.70, 144.43, 165.01, 165.33; ESI-HRMS $m / z$ calcd for $\mathrm{C}_{25} \mathrm{H}_{30} \mathrm{IN}_{2} \mathrm{O}_{3}\left(\mathrm{M}+\mathrm{H}^{+}\right)$: 533.1301; found: 533.1220.

m-Ethynylpyridine Dimer 2. To a toluene $(80 \mathrm{~mL})$ suspension of $\mathrm{NaH}(110 \mathrm{mg}, 2.7 \mathrm{mmol}$; commercial $60 \%$ dispersion was washed thoroughly with hexane prior to use) was added $2 \mathrm{e}(0.71 \mathrm{~g}, 1.3 \mathrm{mmol})$. The reaction mixture was refluxed for $30 \mathrm{~min}$ and evaporated. The evaporated residue was subjected to silica gel column chromatography (eluent; $\left.\mathrm{CH}_{2} \mathrm{Cl}_{2}\right)$ to afford $2(0.28 \mathrm{~g}, 44 \%)$ as dark brown solid. Mp 124-127 ${ }^{\circ} \mathrm{C}$; IR (KBr) 3182, 2950, 2870, 2104, 1582, 1529, $1204 \mathrm{~cm}^{-1} ;{ }^{1} \mathrm{H} \mathrm{NMR}\left(\mathrm{CDCl}_{3}, 300 \mathrm{MHz}\right) \delta 0.98$ (t, $J=7.4 \mathrm{~Hz}, 3 \mathrm{H}), 0.99$ (t, $J=7.5 \mathrm{~Hz}, 3 \mathrm{H}), 1.42-1.52$ (m, $4 \mathrm{H}), 1.72-1.84$ (m, $4 \mathrm{H}), 3.12$ (s, $1 \mathrm{H}), 3.99$ (t, $J$ $=6.6 \mathrm{~Hz}, 2 \mathrm{H}), 4.02(\mathrm{t}, J=6.6 \mathrm{~Hz}, 2 \mathrm{H}), 6.99(\mathrm{~d}, J=2.1 \mathrm{~Hz}, 1 \mathrm{H}), 7.11(\mathrm{~d}, J=2.1 \mathrm{~Hz}, 1 \mathrm{H}), 7.12(\mathrm{~d}, J=2.1$ $\mathrm{Hz}, 1 \mathrm{H}), 7.24(\mathrm{~d}, J=2.1 \mathrm{~Hz}, 1 \mathrm{H}) ;{ }^{13} \mathrm{C} \mathrm{NMR}\left(\mathrm{CDCl}_{3}, 75 \mathrm{MHz}\right) \delta 14.00,19.33,19.36,30.96,30.99,68.59$, S7 
$68.72,77.39,82.39,114.14,114.25,114.62,114.82,121.68,143.73,143.85,165.05,165.38 ;$ ESI-HRMS $m / z$ calcd for $\mathrm{C}_{22} \mathrm{H}_{23} \mathrm{IN}_{2} \mathrm{NaO}_{2}\left(\mathrm{M}+\mathrm{Na}^{+}\right)$: 497.0702; found: 497.0806.

$\boldsymbol{m}$-Ethynylpyridine Trimer 3. To a toluene $(45 \mathrm{~mL})$ suspension of $\mathrm{NaH}(64 \mathrm{mg}, 1.6 \mathrm{mmol}$; commercial $60 \%$ dispersion was washed thoroughly with hexane prior to use) was added $3 \mathbf{e}$ ( $548 \mathrm{mg}, 0.78 \mathrm{mmol})$. The reaction mixture was refluxed for $30 \mathrm{~min}$ and evaporated. The evaporated residue was subjected to silica gel column chromatography (eluent; $\mathrm{CH}_{2} \mathrm{Cl}_{2}$ : hexane $\left.=1: 2\right)$ to afford $3(0.18 \mathrm{~g}, 35 \%)$ as brownish solid. Mp 121-123 ${ }^{\circ} \mathrm{C}$; IR (nujol) 1580, 1560, $1205 \mathrm{~cm}^{-1} ;{ }^{1} \mathrm{H}$ NMR $\left(\mathrm{CDCl}_{3}, 300 \mathrm{MHz}\right) \delta 0.95-1.02(\mathrm{~m}, 9 \mathrm{H})$, 1.43-1.54 (m, $6 \mathrm{H}), 1.73-1.83(\mathrm{~m}, 6 \mathrm{H}), 3.14(\mathrm{~s}, 1 \mathrm{H}), 3.97-4.05(\mathrm{~m}, 6 \mathrm{H}), 6.99(\mathrm{~d}, J=2.1 \mathrm{~Hz}, 1 \mathrm{H}), 7.11(\mathrm{~d}$, $J=2.4 \mathrm{~Hz}, 1 \mathrm{H}), 7.13-7.14(\mathrm{~m}, 3 \mathrm{H}), 7.24(\mathrm{~d}, J=2.1 \mathrm{~Hz}, 1 \mathrm{H}) ;{ }^{13} \mathrm{C} \mathrm{NMR}\left(\mathrm{CDCl}_{3}, 75 \mathrm{MHz}\right) \delta 13.95,19.25$, $19.28,30.89,68.50,68.63,77.35,82.35,86.63,87.30,87.46,88.27,114.11,114.58,114.64,114.69,117.75$, 121.50, 143.60, 143.63, 143.68, 143.86, 143.92, 164.93, 165.30; ESI-HRMS $m / z$ calcd for $\mathrm{C}_{33} \mathrm{H}_{35} \mathrm{IN}_{3} \mathrm{O}_{3}(\mathrm{M}+$ $\mathrm{H}^{+}$): 648.1723; found: 648.1691 .

TBDMS-protected $\boldsymbol{m}$-Ethynylpyridine Tetramer $4 \mathbf{h}$. To an $i-\operatorname{Pr}_{2} \mathrm{NH}(10 \mathrm{~mL})$ and $\mathrm{DMF}(2 \mathrm{~mL})$ suspension of $\mathrm{Pd}_{2}(\mathrm{dba}){ }_{3} \cdot \mathrm{CHCl}_{3}(8.4 \mathrm{mg}, 8.2 \mu \mathrm{mol}), \mathrm{PPh}_{3}(8.6 \mathrm{mg}, 33 \mu \mathrm{mol})$, and $\mathrm{CuI}(0.8 \mathrm{mg}, 4.1 \mu \mathrm{mol})$ were added $\mathbf{1 b}$ (262 mg, $0.65 \mathrm{mmol})$ and $3 \mathrm{~g}(94 \mathrm{mg}, 0.14 \mathrm{mmol})$ subsequently. The mixture was stirred for $2 \mathrm{~h}$ at room temperature and concentrated. The concentrated residue was diluted with $\mathrm{Et}_{2} \mathrm{O}$ and filtered off insoluble salts. The filtrate was evaporated and subjected to silica gel column chromatography (eluent; $\mathrm{CH}_{2} \mathrm{Cl}_{2}:$ hexane $\left.=1: 1\right)$ to afford recovered $\mathbf{1 b}(185 \mathrm{mg}, 88 \%$ recovery $)$ followed by $\mathbf{4 h}(118 \mathrm{mg}, 88 \%$ based

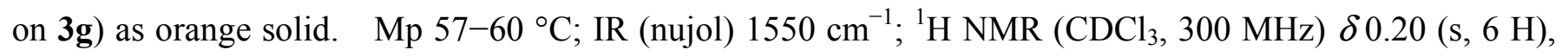
0.96-1.04 (m, $21 \mathrm{H}), 1.44-1.53(\mathrm{~m}, 8 \mathrm{H}), 1.73-1.83(\mathrm{~m}, 8 \mathrm{H}), 3.97-4.05(\mathrm{~m}, 8 \mathrm{H}), 6.97(\mathrm{~d}, J=2.1 \mathrm{~Hz}, 1 \mathrm{H})$, $7.11-7.15(\mathrm{~m}, 6 \mathrm{H}), 7.24(\mathrm{~d}, J=2.1 \mathrm{~Hz}, 1 \mathrm{H}) ;{ }^{13} \mathrm{C} \mathrm{NMR}\left(\mathrm{CDCl}_{3}, 75 \mathrm{MHz}\right) \delta-4.44,14.00,16.96,19.29$, $19.33,26.41,30.94,31.01,68.43,68.66,86.70,87.30,87.38,87.56,87.73,88.32,93.56,104.01,113.88$, $114.62,114.75,114.81,117.78,121.60,143.64,143.75,143.81,143.87,143.99,144.05,144.54,164.97$, 165.20, 165.35; ESI-HRMS $m / z$ calcd for $\mathrm{C}_{50} \mathrm{H}_{60} \mathrm{IN}_{4} \mathrm{O}_{4} \mathrm{Si}\left(\mathrm{M}+\mathrm{H}^{+}\right)$: 935.3429; found: 935.3373 .

$\boldsymbol{m}$-Ethynylpyridine Tetramer 4 . To a THF $(1 \mathrm{~mL})$ solution of $\mathbf{4 h}(118 \mathrm{mg}, 0.126 \mathrm{mmol})$ were added $n$ - $\mathrm{Bu}_{4} \mathrm{NF}(1.0 \mathrm{M}$ THF solution, $0.126 \mathrm{~mL}, 0.126 \mathrm{mmol})$ and a few drops of $\mathrm{H}_{2} \mathrm{O}$. The mixture was stirred 
for $1.5 \mathrm{~h}$ at room temperature, concentrated, and extracted with $\mathrm{CHCl}_{3}$. The extract was washed with $\mathrm{H}_{2} \mathrm{O}$, dried over $\mathrm{K}_{2} \mathrm{CO}_{3}$, and evaporated. The evaporated residue was subjected to silica gel column chromatography (eluent; $\mathrm{CH}_{2} \mathrm{Cl}_{2}$ : hexane $\left.=1: 1\right)$ to afford $4(83 \mathrm{mg}, 80 \%)$ as brown oil. IR (nujol) 1575 , $1210 \mathrm{~cm}^{-1} ;{ }^{1} \mathrm{H} \mathrm{NMR}\left(\mathrm{CDCl}_{3}, 300 \mathrm{MHz}\right) \delta 0.97-1.01(\mathrm{~m}, 12 \mathrm{H}), 1.45-1.52(\mathrm{~m}, 8 \mathrm{H}), 1.76-1.83(\mathrm{~m}, 8 \mathrm{H})$, $3.13(\mathrm{~s}, 1 \mathrm{H}), 3.97-4.05(\mathrm{~m}, 8 \mathrm{H}), 7.00(\mathrm{~d}, J=2.5 \mathrm{~Hz}, 1 \mathrm{H}), 7.12(\mathrm{~d}, J=2.5 \mathrm{~Hz}, 1 \mathrm{H}), 7.14-7.15(\mathrm{~m}, 5 \mathrm{H})$, $7.25(\mathrm{~d}, J=2.5 \mathrm{~Hz}, 1 \mathrm{H}) ;{ }^{13} \mathrm{C} \mathrm{NMR}\left(\mathrm{CDCl}_{3}, 75 \mathrm{MHz}\right) \delta 13.72,13.74,19.93,19.07,29.71,30.69,30.70$, $30.72,68.37,68.48,68.49,68.52,82.24,87.26,87.38,88.71,114.07,114.55,114.61,114.64,114.72,117.72$, 143.64, 143.74, 143.86, 143.91, 143.92, 143.94, 144.38, 165.36, 165.38, 165.41; ESI-HRMS m/z calcd for $\mathrm{C}_{44} \mathrm{H}_{46} \mathrm{IN}_{4} \mathrm{O}_{4}\left(\mathrm{M}+\mathrm{H}^{+}\right): 821.2564$; found: 821.2392 .

TBDMS-protected $\boldsymbol{m}$-Ethynylpyridine Pentamer $5 \mathbf{h}$. To an $i$ - $\operatorname{Pr}_{2} \mathrm{NH}(5 \mathrm{~mL})$ and $\mathrm{DMF}(1 \mathrm{~mL})$ suspension of $\mathrm{Pd}_{2}(\mathrm{dba}){ }_{3} \cdot \mathrm{CHCl}_{3}(2.7 \mathrm{mg}, 2.6 \mu \mathrm{mol}), \mathrm{PPh}_{3}(2.8 \mathrm{mg}, 11 \mu \mathrm{mol})$, and $\mathrm{CuI}(0.3 \mathrm{mg}, 1.3 \mu \mathrm{mol})$ were added $\mathbf{2 b}(122 \mathrm{mg}, 0.212 \mathrm{mmol})$ and $\mathbf{3 g}(27 \mathrm{mg}, 0.041 \mathrm{mmol})$ subsequently. The mixture was stirred for $2 \mathrm{~h}$ at room temperature and concentrated. The concentrated residue was diluted with $\mathrm{Et}_{2} \mathrm{O}$ and filtered off insoluble salts. The filtrate was evaporated and subjected to silica gel column chromatography (eluent; $\mathrm{CH}_{2} \mathrm{Cl}_{2}$ : hexane $\left.=1: 1\right)$ to afford recovered $\mathbf{2 b}$ followed by $\mathbf{5 h}(42 \mathrm{mg}, 91 \%$ based on $\mathbf{3 g})$ as brownish solid. Mp 69-71 ${ }^{\circ} \mathrm{C}$; IR (nujol) $1550 \mathrm{~cm}^{-1} ;{ }^{1} \mathrm{H}$ NMR $\left(\mathrm{CDCl}_{3}, 300 \mathrm{MHz}\right) \delta 0.20(\mathrm{~s}, 6 \mathrm{H}), 0.94-1.04(\mathrm{~m}, 24 \mathrm{H})$, 1.43-1.54 (m, $10 \mathrm{H}), 1.73-1.84(\mathrm{~m}, 10 \mathrm{H}), 3.97-4.06(\mathrm{~m}, 10 \mathrm{H}), 6.98(\mathrm{~d}, J=2.4 \mathrm{~Hz}, 1 \mathrm{H}), 7.11-7.16(\mathrm{~m}, 8$ $\mathrm{H}), 7.25(\mathrm{~d}, J=2.4 \mathrm{~Hz}, 1 \mathrm{H}) ;{ }^{13} \mathrm{C} \mathrm{NMR}\left(\mathrm{CDCl}_{3}, 75 \mathrm{MHz}\right) \delta-4.49,13.95,16.92,19.28,26.36,30.89,30.96$, $68.38,68.59,68.82,86.65,87.27,87.38,87.47,87.67,88.25,93.49,103.98,113.85,114.22,114.60,114.67$, $114.78,117.75,121.52,143.60,143.70,143.76,143.83,143.92,143.97,144.51,164.93,165.15,165.28$; ESI-HRMS $m / z$ calcd for $\mathrm{C}_{61} \mathrm{H}_{71} \mathrm{IN}_{5} \mathrm{O}_{5} \mathrm{Si}\left(\mathrm{M}+\mathrm{H}^{+}\right)$: 1108.4269 ; found: 1108.4261 .

m-Ethynylpyridine Pentamer 5. To a THF $(1 \mathrm{~mL})$ solution of $\mathbf{5 h}(42 \mathrm{mg}, 0.038 \mathrm{mmol})$ were added $n$ - $\mathrm{Bu}_{4} \mathrm{NF}(1.0 \mathrm{M}$ THF solution, $0.038 \mathrm{~mL}, 0.038 \mathrm{mmol})$ and a few drops of $\mathrm{H}_{2} \mathrm{O}$. The mixture was stirred for $1.5 \mathrm{~h}$ at room temperature, concentrated, and extracted with $\mathrm{CHCl}_{3}$. The $\mathrm{CHCl}_{3}$ extract was washed with $\mathrm{H}_{2} \mathrm{O}$, dried over $\mathrm{K}_{2} \mathrm{CO}_{3}$, and evaporated. The evaporated residue was subjected to silica gel column chromatography (eluent; $\mathrm{CH}_{2} \mathrm{Cl}_{2}$ : hexane $\left.=1: 1\right)$ to afford $5(30 \mathrm{mg}, 80 \%)$ as brownish solid. IR (nujol) 
$1565 \mathrm{~cm}^{-1} ;{ }^{1} \mathrm{H}$ NMR $\left(\mathrm{CDCl}_{3}, 500 \mathrm{MHz}\right) \delta 0.97-1.01(\mathrm{~m}, 15 \mathrm{H}), 1.45-1.54(\mathrm{~m}, 10 \mathrm{H}), 1.76-1.84(\mathrm{~m}, 10 \mathrm{H})$, $3.13(\mathrm{~s}, 1 \mathrm{H}), 3.98-4.05(\mathrm{~m}, 10 \mathrm{H}), 7.00(\mathrm{~d}, J=2.5 \mathrm{~Hz}, 1 \mathrm{H}), 7.12(\mathrm{~d}, J=2.5 \mathrm{~Hz}, 1 \mathrm{H}), 7.14-7.16(\mathrm{~m}, 7 \mathrm{H})$, $7.25(\mathrm{~d}, J=2.5 \mathrm{~Hz}, 1 \mathrm{H}) ;{ }^{13} \mathrm{C} \mathrm{NMR}\left(\mathrm{CDCl}_{3}, 75 \mathrm{MHz}\right) \delta 13.71,13.74,19.04,19.07,30.69,30.70,30.72$, $68.36,68.47,68.50,68.52,77.20,82.25,86.55,87.26,87.28,87.31,87.33,87.35,87.38,88.71,114.07$, $114.55,114.63,114.71,117.72,121.52,143.64,143.74,143.89,143.92,165.01,165.35,165.37,165.38$; ESI-HRMS $m / z$ calcd for $\mathrm{C}_{55} \mathrm{H}_{57} \mathrm{IN}_{5} \mathrm{O}_{5}\left(\mathrm{M}+\mathrm{H}^{+}\right)$: 994.3404; found: 994.3294.

TBDMS-protected $\boldsymbol{m}$-Ethynylpyridine 12 -mer $12 \mathbf{h}$. To an $i-\operatorname{Pr}_{2} \mathrm{NH}(100 \mathrm{~mL})$ and $\mathrm{DMF}(10 \mathrm{~mL})$ suspension of $\mathrm{Pd}_{2}(\mathrm{dba})_{3} \cdot \mathrm{CHCl}_{3}(13 \mathrm{mg}, 12 \mu \mathrm{mol}), \mathrm{PPh}_{3}(13 \mathrm{mg}, 49 \mu \mathrm{mol})$, and $\mathrm{CuI}(1.2 \mathrm{mg}, 6.2 \mu \mathrm{mol})$ were added $6(0.29 \mathrm{~g}, 0.25 \mathrm{mmol})$ and $6 \mathrm{~h}(1.6 \mathrm{~g}, 1.2 \mathrm{mmol})$ subsequently. The mixture was stirred for $2 \mathrm{~h}$ at room temperature and concentrated. The concentrated residue was subjected to silica gel column chromatography (eluent; $\left.\mathrm{CH}_{2} \mathrm{Cl}_{2}: \mathrm{MeOH}=100: 1\right)$ to afford recovered $\mathbf{6 h}(1.1 \mathrm{~g}, 83 \%$ recovery) followed by $\mathbf{1 2 h}(0.45 \mathrm{~g}, 79 \%$ based on $\mathbf{6})$ as brownish solid. Mp $188-190{ }^{\circ} \mathrm{C}$; IR (KBr) 2955, 2870, 1583, 1548, 1186, $1037 \mathrm{~cm}^{-1}$; ${ }^{1} \mathrm{H}$ NMR $\left(\mathrm{CDCl}_{3}, 300 \mathrm{MHz}\right) \delta 0.20(\mathrm{~s}, 6 \mathrm{H}), 0.97-1.03(\mathrm{~m}, 45 \mathrm{H}), 1.44-1.57(\mathrm{~m}, 24 \mathrm{H})$, $1.74-1.86(\mathrm{~m}, 24 \mathrm{H}), 3.97-4.06(\mathrm{~m}, 24 \mathrm{H}), 6.97(\mathrm{~d}, J=2.7 \mathrm{~Hz}, 1 \mathrm{H}), 7.11-7.19(\mathrm{~m}, 22 \mathrm{H}), 7.24(\mathrm{~d}, J=2.1$ $\mathrm{Hz}, 1 \mathrm{H}) ;{ }^{13} \mathrm{C} \mathrm{NMR}\left(\mathrm{CDCl}_{3}, 75 \mathrm{MHz}\right) \delta 14.02,19.36,26.43,30.98,68.66,87.51,114.75,143.97,165.38$; ESI-HRMS $m / z$ calcd for $\mathrm{C}_{138} \mathrm{H}_{147} \mathrm{IN}_{12} \mathrm{NaO}_{12} \mathrm{Si}\left(\mathrm{M}+\mathrm{Na}^{+}\right)$: 2341.9973 ; found: 2341.9428 .

$\boldsymbol{m}$-Ethynylpyridine 12-mer $\mathbf{1 2}$. To a THF $(1 \mathrm{~mL})$ solution of $\mathbf{1 2 h}(120 \mathrm{mg}, 0.052 \mathrm{mmol})$ were added $n$ - $\mathrm{Bu}_{4} \mathrm{NF}(1.0 \mathrm{M}$ THF solution, $0.052 \mathrm{~mL}, 0.052 \mathrm{mmol})$ and a few drops of $\mathrm{H}_{2} \mathrm{O}$. The mixture was stirred for $1.5 \mathrm{~h}$ at room temperature, concentrated, and extracted with $\mathrm{CHCl}_{3}$. The $\mathrm{CHCl}_{3}$ extract was washed with $\mathrm{H}_{2} \mathrm{O}$, dried over $\mathrm{K}_{2} \mathrm{CO}_{3}$, and evaporated. The evaporated residue was subjected to silica gel column chromatography (eluent; $\left.\mathrm{CH}_{2} \mathrm{Cl}_{2}: \mathrm{MeOH}=50: 1\right)$ to afford $12(109 \mathrm{mg}, 96 \%)$ as brownish solid. $\mathrm{Mp}$ $113-116^{\circ} \mathrm{C}$; IR (KBr) 2930, 2870, 1582, 1548, 1192, $1037 \mathrm{~cm}^{-1} ;{ }^{1} \mathrm{H}$ NMR $\left(\mathrm{CDCl}_{3}, 300 \mathrm{MHz}\right) \delta 1.00(\mathrm{t}, J=$ $7.4 \mathrm{~Hz}, 36 \mathrm{H}), 1.44-1.57(\mathrm{~m}, 24 \mathrm{H}), 1.74-1.86(\mathrm{~m}, 24 \mathrm{H}), 3.13(\mathrm{~s}, 1 \mathrm{H}), 3.97-4.06(\mathrm{~m}, 24 \mathrm{H}), 7.00(\mathrm{~d}, J=2.7$ $\mathrm{Hz}, 1 \mathrm{H}), 7.12-7.19(\mathrm{~m}, 22 \mathrm{H}), 7.24(\mathrm{~d}, J=2.1 \mathrm{~Hz}, 1 \mathrm{H}) ;{ }^{13} \mathrm{C} \mathrm{NMR}\left(\mathrm{CDCl}_{3}, 75 \mathrm{MHz}\right) \delta 14.02,19.36,30.96$, 68.66, 87.51, 114.75, 143.97, 165.38; ESI-HRMS $m / z$ calcd for $\mathrm{C}_{132} \mathrm{H}_{134} \mathrm{IN}_{12} \mathrm{O}_{12}\left(\mathrm{M}+\mathrm{H}^{+}\right)$: 2205.9289; found: 2205.8716 . 
TBDMS-protected $\boldsymbol{m}$-Ethynylpyridine 18-mer $18 \mathrm{~h}$. To an $i$ - $\operatorname{Pr}_{2} \mathrm{NH}(30 \mathrm{~mL})$ and DMF $(20 \mathrm{~mL})$ suspension of $\mathrm{Pd}_{2}(\mathrm{dba})_{3} \cdot \mathrm{CHCl}_{3}(2.1 \mathrm{mg}, 2 \mu \mathrm{mol}), \mathrm{PPh}_{3}(2.1 \mathrm{mg}, 8 \mu \mathrm{mol})$, and $\mathrm{CuI}(0.2 \mathrm{mg}, 1 \mu \mathrm{mol})$ were added $\mathbf{6}(47 \mathrm{mg}, 0.040 \mathrm{mmol})$ and $\mathbf{1 2 h}(390 \mathrm{mg}, 0.17 \mathrm{mmol})$ subsequently. The mixture was stirred for $3 \mathrm{~h}$ at room temperature and concentrated. The concentrated residue was subjected to silica gel column chromatography (eluent; $\left.\mathrm{CH}_{2} \mathrm{Cl}_{2}: \mathrm{MeOH}=50: 1\right)$ to afford recovered $\mathbf{1 2} \mathbf{h}$ ( $246 \mathrm{mg}, 79 \%$ recovery) followed by $\mathbf{1 8 h}\left(115 \mathrm{mg}, 85 \%\right.$ based on 6) as brownish solid. $\mathrm{Mp} 196-199{ }^{\circ} \mathrm{C}$; IR (neat) $1589,1543,1211 \mathrm{~cm}^{-1} ;{ }^{1} \mathrm{H}$ $\operatorname{NMR}\left(\mathrm{CDCl}_{3}, 300 \mathrm{MHz}\right) \delta 0.20(\mathrm{~s}, 6 \mathrm{H}), 0.97-1.03(\mathrm{~m}, 63 \mathrm{H}), 1.44-1.54(\mathrm{~m}, 36 \mathrm{H}), 1.76-1.86(\mathrm{~m}, 36 \mathrm{H})$, 3.99-4.06 (m, $36 \mathrm{H}), 6.97(\mathrm{~d}, J=2.1 \mathrm{~Hz}, 1 \mathrm{H}), 7.12-7.17(\mathrm{~m}, 34 \mathrm{H}), 7.24(\mathrm{~d}, J=2.1 \mathrm{~Hz}, 1 \mathrm{H}) ;{ }^{13} \mathrm{C}$ NMR $\left(\mathrm{CDCl}_{3}, 75 \mathrm{MHz}\right) \delta 14.04,19.36,30.98,68.66,87.51,114.77,143.97,165.38$; ESI-HRMS $\mathrm{m} / z$ calcd for $\mathrm{C}_{204} \mathrm{H}_{213} \mathrm{IN}_{18} \mathrm{NaO}_{18} \mathrm{Si}\left(\mathrm{M}+3+\mathrm{Na}^{+}\right): 3382.5084$; found: 3382.5350 .

$\boldsymbol{m}$-Ethynylpyridine $\mathbf{1 8}$-mer $\mathbf{1 8}$. To a THF $(1 \mathrm{~mL})$ and $\mathrm{CHCl}_{3}(1 \mathrm{~mL})$ solution of $\mathbf{1 8 h}(67 \mathrm{mg}, 20 \mu \mathrm{mol})$ were added $n$-Bu $\mathrm{Bu}_{4} \mathrm{NF}(1.0 \mathrm{M}$ THF solution, $20 \mu \mathrm{L}, 20 \mu \mathrm{mol})$ and a few drops of $\mathrm{H}_{2} \mathrm{O}$. The mixture was stirred for $1.5 \mathrm{~h}$ at room temperature, concentrated, and extracted with $\mathrm{CHCl}_{3}$. The $\mathrm{CHCl}_{3}$ extract was washed with $\mathrm{H}_{2} \mathrm{O}$, dried over $\mathrm{K}_{2} \mathrm{CO}_{3}$, and evaporated. The evaporated residue was subjected to silica gel column chromatography (eluent; $\left.\mathrm{CH}_{2} \mathrm{Cl}_{2}: \mathrm{MeOH}=50: 1\right)$ to afford $18(56 \mathrm{mg}, 86 \%)$ as brownish solid. Mp 205-209 ${ }^{\circ} \mathrm{C}$; IR (KBr) 2955, 2870, 1583, 1547, 1189, $1036 \mathrm{~cm}^{-1} ;{ }^{1} \mathrm{H}$ NMR $\left(\mathrm{CDCl}_{3}, 300 \mathrm{MHz}\right) \delta 1.00(\mathrm{t}$, $J=7.4 \mathrm{~Hz}, 54 \mathrm{H}), 1.44-1.57(\mathrm{~m}, 36 \mathrm{H}), 1.74-1.86(\mathrm{~m}, 36 \mathrm{H}), 3.13(\mathrm{~s}, 1 \mathrm{H}), 3.97-4.06(\mathrm{~m}, 36 \mathrm{H}), 7.00(\mathrm{~d}, J=$ $2.7 \mathrm{~Hz}, 1 \mathrm{H}), 7.12-7.19(\mathrm{~m}, 34 \mathrm{H}), 7.24(\mathrm{~d}, J=2.4 \mathrm{~Hz}, 1 \mathrm{H}) ;{ }^{13} \mathrm{C} \mathrm{NMR}\left(\mathrm{CDCl}_{3}, 75 \mathrm{MHz}\right) \delta 14.02,19.36$, 30.98, 68.66, 87.51, 114.75, 143.97, 165.38; ESI-HRMS $m / z$ calcd for $\mathrm{C}_{198} \mathrm{H}_{199} \mathrm{IN}_{18} \mathrm{NaO}_{18}\left(\mathrm{M}+2+\mathrm{H}^{+}\right)$: 3268.4219; found: 3268.4072 .

TBDMS-protected $\boldsymbol{m}$-Ethynylpyridine 24-mer $24 \mathbf{h}$. To an $i$ - $\operatorname{Pr}_{2} \mathrm{NH}(30 \mathrm{~mL})$ and DMF $(30 \mathrm{~mL})$ suspension of $\mathrm{Pd}_{2}(\mathrm{dba})_{3} \cdot \mathrm{CHCl}_{3}(0.75 \mathrm{mg}, 0.73 \mu \mathrm{mol}), \mathrm{PPh}_{3}(0.76 \mathrm{mg}, 2.9 \mu \mathrm{mol})$, and $\mathrm{CuI}(0.1 \mathrm{mg}, 0.36$ $\mu \mathrm{mol})$ were added $12(32 \mathrm{mg}, 15 \mu \mathrm{mol})$ and $\mathbf{1 2 h}(135 \mathrm{mg}, 58 \mu \mathrm{mol})$ subsequently. The mixture was stirred for $2 \mathrm{~h}$ at room temperature and concentrated. The concentrated residue was subjected to silica gel column chromatography (eluent; $\mathrm{CH}_{2} \mathrm{Cl}_{2}: \mathrm{MeOH}=50: 1$ to $\left.25: 1\right)$ to afford recovered $\mathbf{1 2 h}(100 \mathrm{mg}, 83 \%$ recovery) followed by $\mathbf{2 4 h}\left(26 \mathrm{mg}, 41 \%\right.$ based on 12) as brown solid. $\mathrm{Mp} 176-178{ }^{\circ} \mathrm{C}$ (dec); IR (nujol) 1590,1535 , 
$1210 \mathrm{~cm}^{-1} ;{ }^{1} \mathrm{H}$ NMR $\left(\mathrm{CDCl}_{3}, 300 \mathrm{MHz}\right) \delta 0.20(\mathrm{~s}, 6 \mathrm{H}), 1.00(\mathrm{~m}, 81 \mathrm{H}), 1.46-1.55(\mathrm{~m}, 48 \mathrm{H}), 1.76-1.85(\mathrm{~m}$, $48 \mathrm{H}), 4.02-4.07(\mathrm{~m}, 48 \mathrm{H}), 6.97(\mathrm{~d}, J=2.1 \mathrm{~Hz}, 1 \mathrm{H}), 7.13-7.17(\mathrm{~m}, 46 \mathrm{H}), 7.24(\mathrm{~d}, J=2.1 \mathrm{~Hz}, 1 \mathrm{H}) ;{ }^{13} \mathrm{C}$ NMR $\left(\mathrm{CDCl}_{3}, 75 \mathrm{MHz}\right) \delta 14.02,19.34,30.96,68.64,87.49,114.75,143.96,165.36$; ESI-HRMS $m / z$ calcd for $\mathrm{C}_{270} \mathrm{H}_{281} \mathrm{IN}_{24} \mathrm{O}_{24} \mathrm{Si}\left(\mathrm{M}+5+2 \mathrm{H}^{+}\right): 2200.5210$; found: 2200.4080 .

m-Ethynylpyridine 24-mer 24. To a THF (1 mL) and $\mathrm{CHCl}_{3}$ (a few drops) solution of $\mathbf{2 4 h}(37 \mathrm{mg}, 8.5$ $\mu \mathrm{mol})$ were added $n$-Bu $\mathrm{BF}_{4}(1.0 \mathrm{M}$ THF solution, $10 \mu \mathrm{L}, 10 \mu \mathrm{mol})$ and a few drops of $\mathrm{H}_{2} \mathrm{O}$. The mixture was stirred for $1.5 \mathrm{~h}$ at room temperature and evaporated. The evaporated residue was subjected to silica gel column chromatography (eluent; $\left.\mathrm{CH}_{2} \mathrm{Cl}_{2}: \mathrm{MeOH}=50: 1\right)$ to afford $24(36 \mathrm{mg}, 100 \%)$ as brownish solid. Mp 162-165 ${ }^{\circ} \mathrm{C}$ (dec); IR (nujol) 1550, $1220 \mathrm{~cm}^{-1} ;{ }^{1} \mathrm{H}$ NMR $\left(\mathrm{CDCl}_{3}, 300 \mathrm{MHz}\right) \delta 1.00(\mathrm{t}, J=7.2 \mathrm{~Hz}, 72 \mathrm{H})$, 1.46-1.55 (m, $48 \mathrm{H}), 1.78-1.84(\mathrm{~m}, 48 \mathrm{H}), 3.13(\mathrm{~s}, 1 \mathrm{H}), 4.04(\mathrm{t}, J=6.3 \mathrm{~Hz}, 48 \mathrm{H}), 7.00(\mathrm{~d}, J=2.1 \mathrm{~Hz}, 1 \mathrm{H})$, 7.13-7.16 (m, $46 \mathrm{H}), 7.25(\mathrm{~d}, J=2.1 \mathrm{~Hz}, 1 \mathrm{H})$; ESI-HRMS $m / z$ calcd for $\mathrm{C}_{264} \mathrm{H}_{267} \mathrm{~N}_{24} \mathrm{O}_{24}\left(\mathrm{M}+4+2 \mathrm{H}^{+}\right)$: 2142.9761; found: 2142.7957 .

Extraction Experiments for Native Saccharides. Saccharide $(2.0 \mathrm{mg})$ was suspended in a $\mathrm{CH}_{2} \mathrm{Cl}_{2}(1.0$ $\mathrm{mL}$ ) solution of $\mathbf{1}_{\text {poly }}\left(1.0 \times 10^{-3} \mathrm{M}\right.$, monomer unit concentration), and the suspension was sonicated for $1 \mathrm{~h}$ at room temperature. After standing for $12 \mathrm{~h}$, the mixture was filtered through a membrane filter $(0.20 \mu \mathrm{m})$, and the filtrate was analyzed by a CD spectrometer $\left(25^{\circ} \mathrm{C}\right.$, light-path length was $\left.1 \mathrm{~mm}\right)$.

\section{References}

(1) Inouye, M.; Miyake, T.; Furusyo, M.; Nakazumi, H. J. Am. Chem. Soc. 1995, 117, 12416-12425.

(2) Rodríguez-Ubis, J. C.; Sedano, R.; Barroso, G.; Juanes, O.; Brunet, E. Helv. Chim. Acta 1997, 80, $86-96$.

(3) Suzuki, H.; Kondo, A.; Inouye, M.; Ogawa, T. Synthesis 1986, 121-122. 
Figures S1, S2, S3 and S4

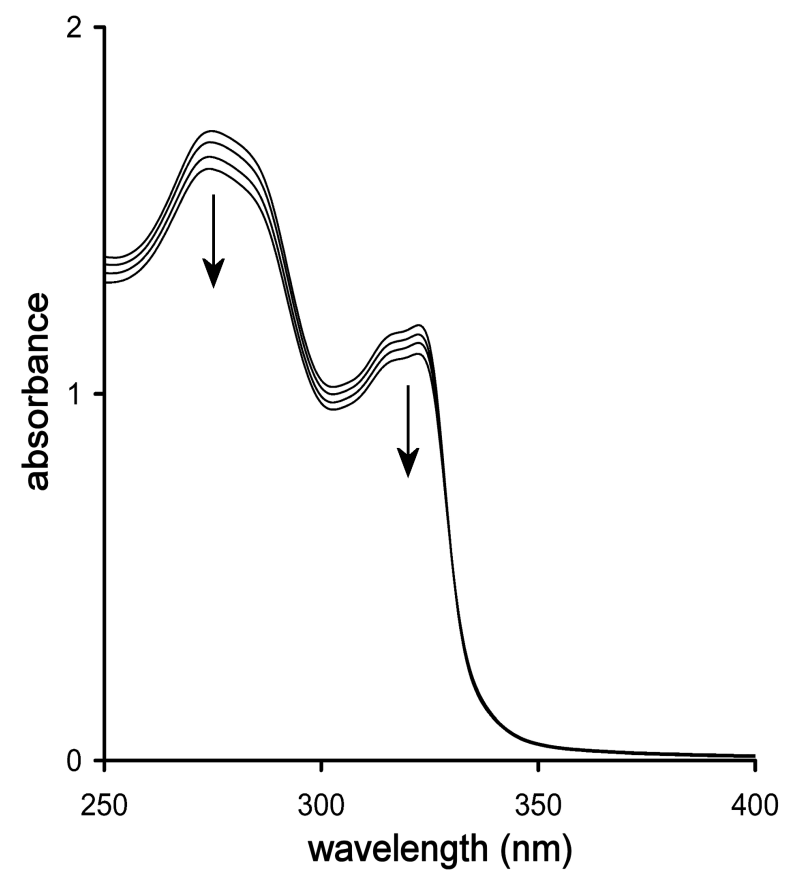

Figure S1. The hypochromism of the absorption spectra of $\mathbf{1}_{\text {poly }}$ by the addition of $\boldsymbol{\beta}$-D-Glc. Conditions: $\mathbf{1}_{\text {poly }}\left(1.0 \times 10^{-3} \mathrm{M}\right.$, monomer unit concentration $) ; \beta$-D-Glc $\left(0-1.8 \times 10^{-3} \mathrm{M}\right) ; \mathrm{CH}_{2} \mathrm{Cl}_{2} ; 25^{\circ} \mathrm{C}$. Light-path length was $1 \mathrm{~mm}$.

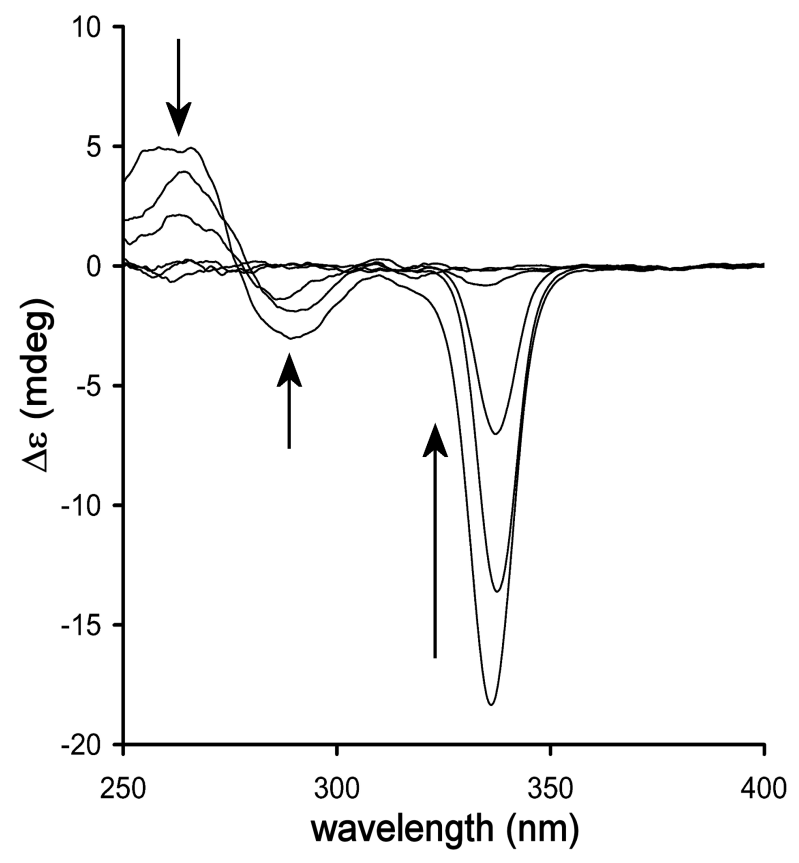

Figure $\mathbf{S 2}$. The quenching of the ICD by the addition of $\mathrm{MeOH}$ to $\mathbf{1}_{\text {poly }}$ with $\boldsymbol{\beta}$-D-Glc in $\mathrm{CH}_{2} \mathrm{Cl}_{2}$. Conditions: $\mathbf{1}_{\text {poly }}\left(1.0 \times 10^{-3} \mathrm{M}\right.$, monomer unit concentration $) ; \beta$-D-Glc $\left(2.0 \times 10^{-3} \mathrm{M}\right) ; \mathrm{CH}_{2} \mathrm{Cl}_{2} / \mathrm{MeOH}$ (100:0, 99:1, 97:3, 95:5, 90:10, and 80:20); $25^{\circ} \mathrm{C}$. Light-path length was $1 \mathrm{~mm}$. 


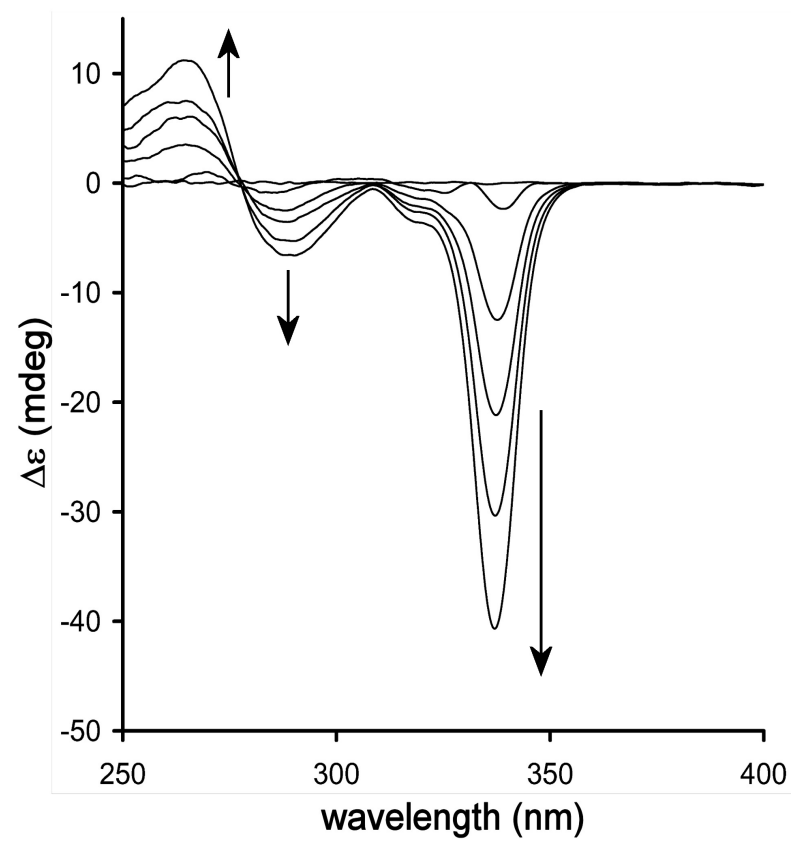

Figure S3. The CD titration of $\beta$-D-Glc to 24 . Conditions: $24\left(4.0 \times 10^{-5} \mathrm{M}\right) ; \boldsymbol{\beta}$-D-Glc $(0-50$ eq); $\mathrm{CH}_{2} \mathrm{Cl}_{2} ; 25{ }^{\circ} \mathrm{C}$. Light-path length was $1 \mathrm{~mm}$.

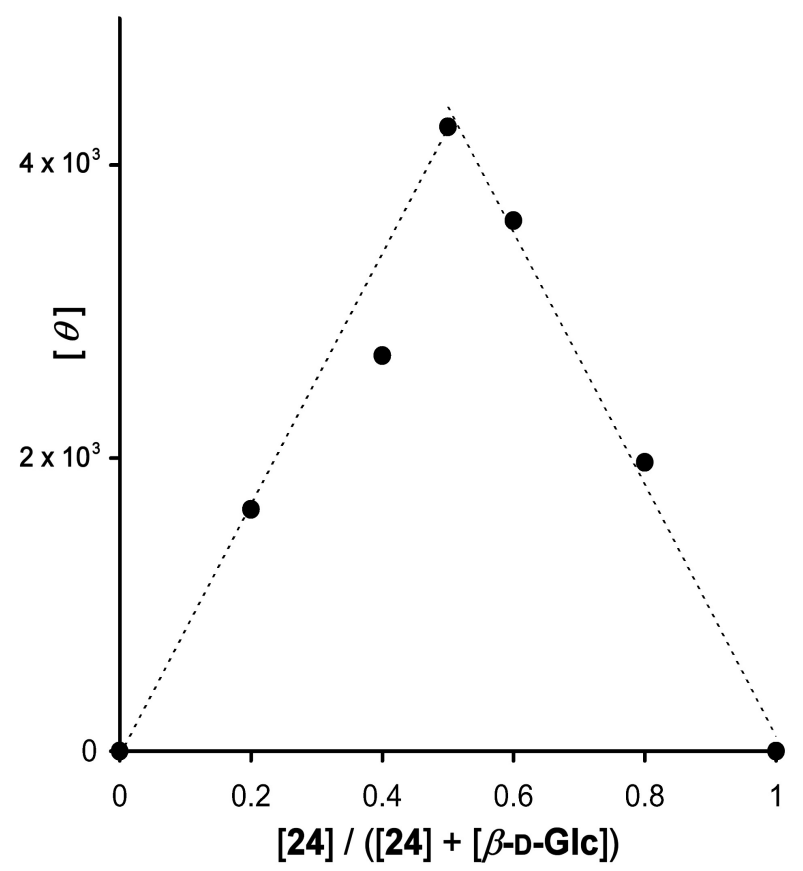

Figure S4. Job's plot for 24 and $\beta$-D-Glc versus the molar ellipticity at $340 \mathrm{~nm}$. Conditions: ([24] + $[\beta$-D-Glc $])=1.0 \times 10^{-3} \mathrm{M} ; \mathrm{CH}_{2} \mathrm{Cl}_{2} ; 25^{\circ} \mathrm{C}$. Light-path length was $1 \mathrm{~mm}$. 
${ }^{1} \mathrm{H}$ NMR spectra for compounds $1,1 \mathrm{~b}-\mathrm{d}, 2,2 \mathrm{~b}, 2 \mathrm{e}, 3,3 \mathrm{~b}, 3 \mathrm{e}-\mathrm{g}, 4,4 \mathrm{~h}, 5,5 \mathrm{~h}, 6,6 \mathrm{~h}, 12,12 \mathrm{~h}, 18,18 \mathrm{~h}, 24$, and 24h
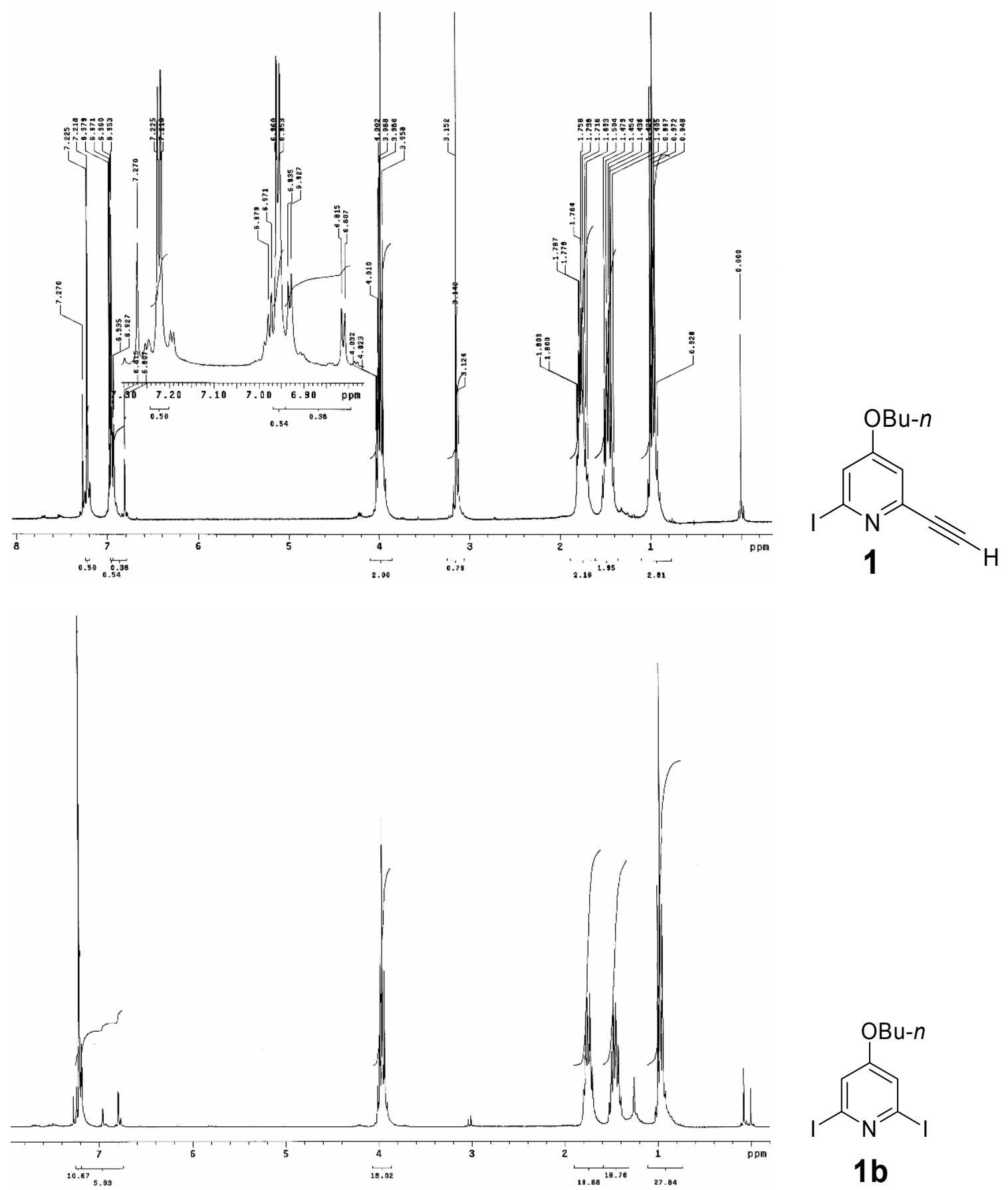

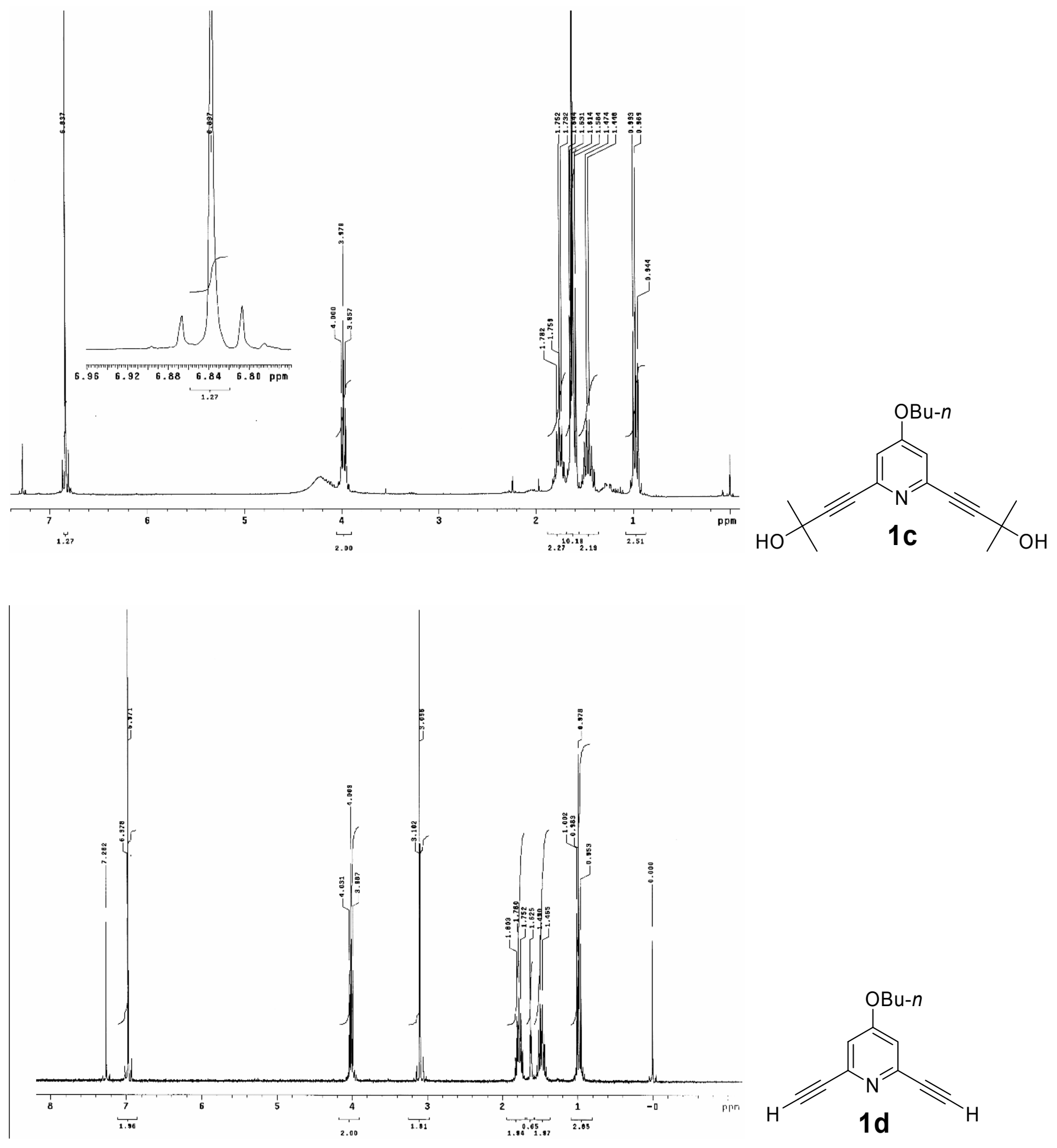

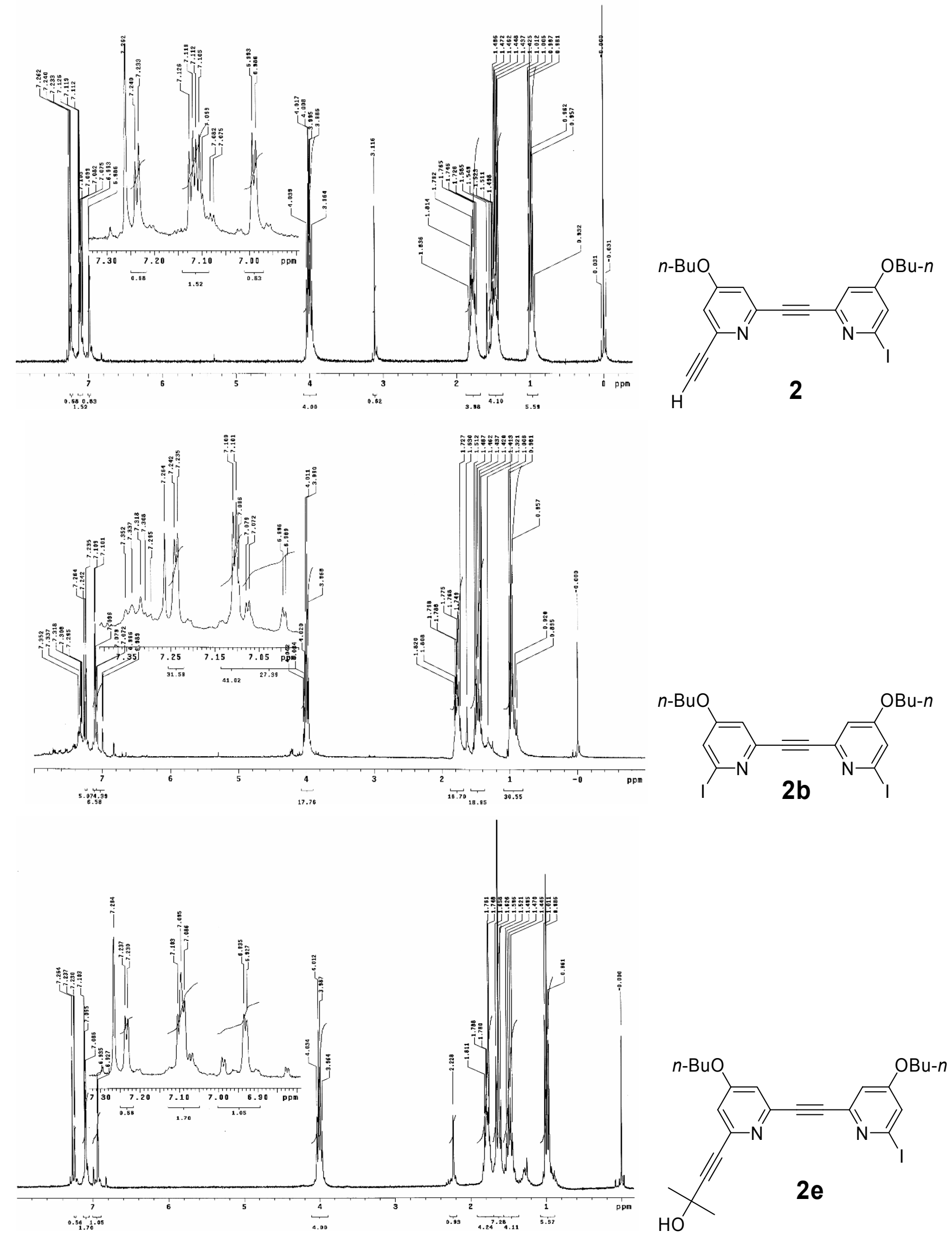

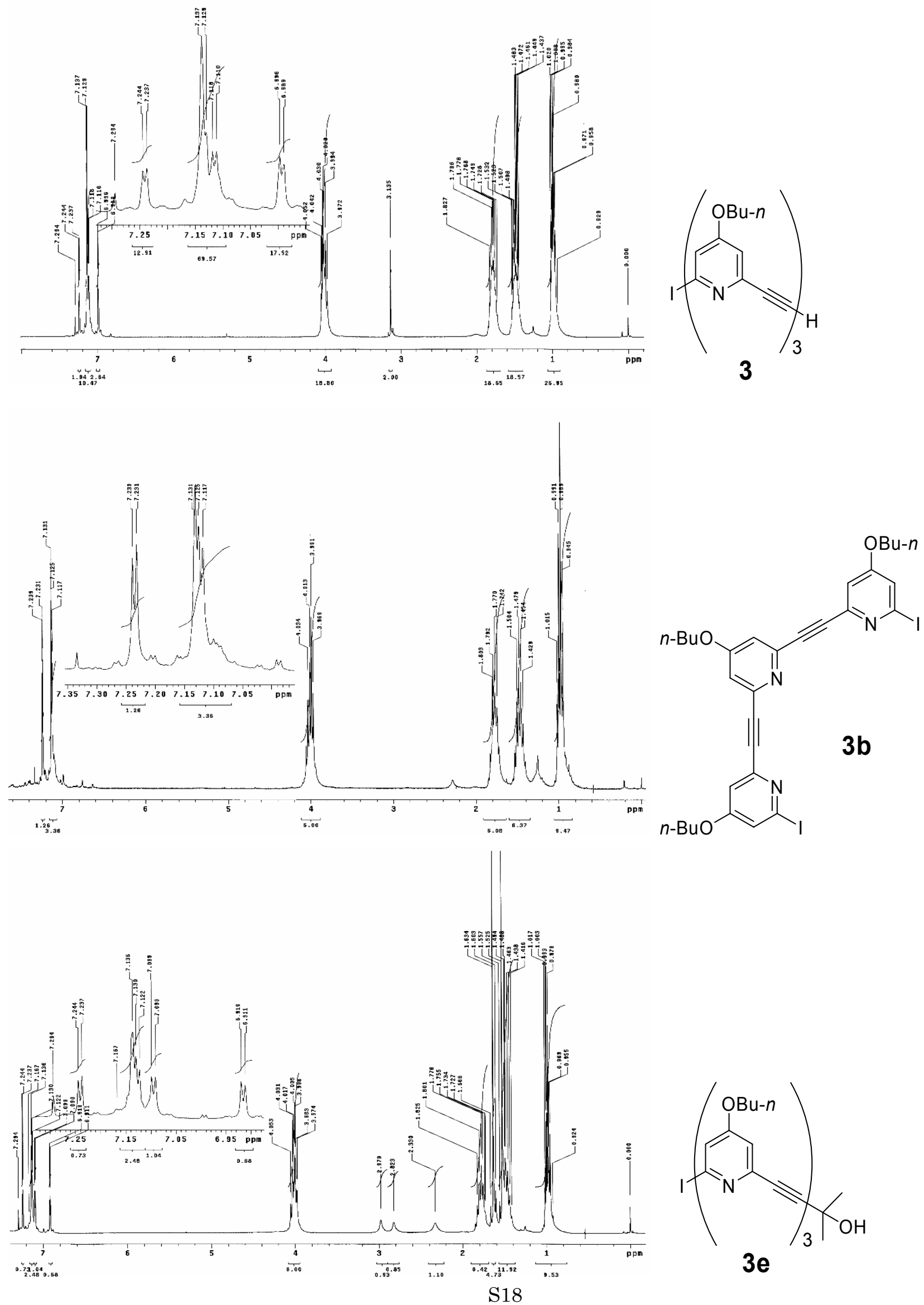

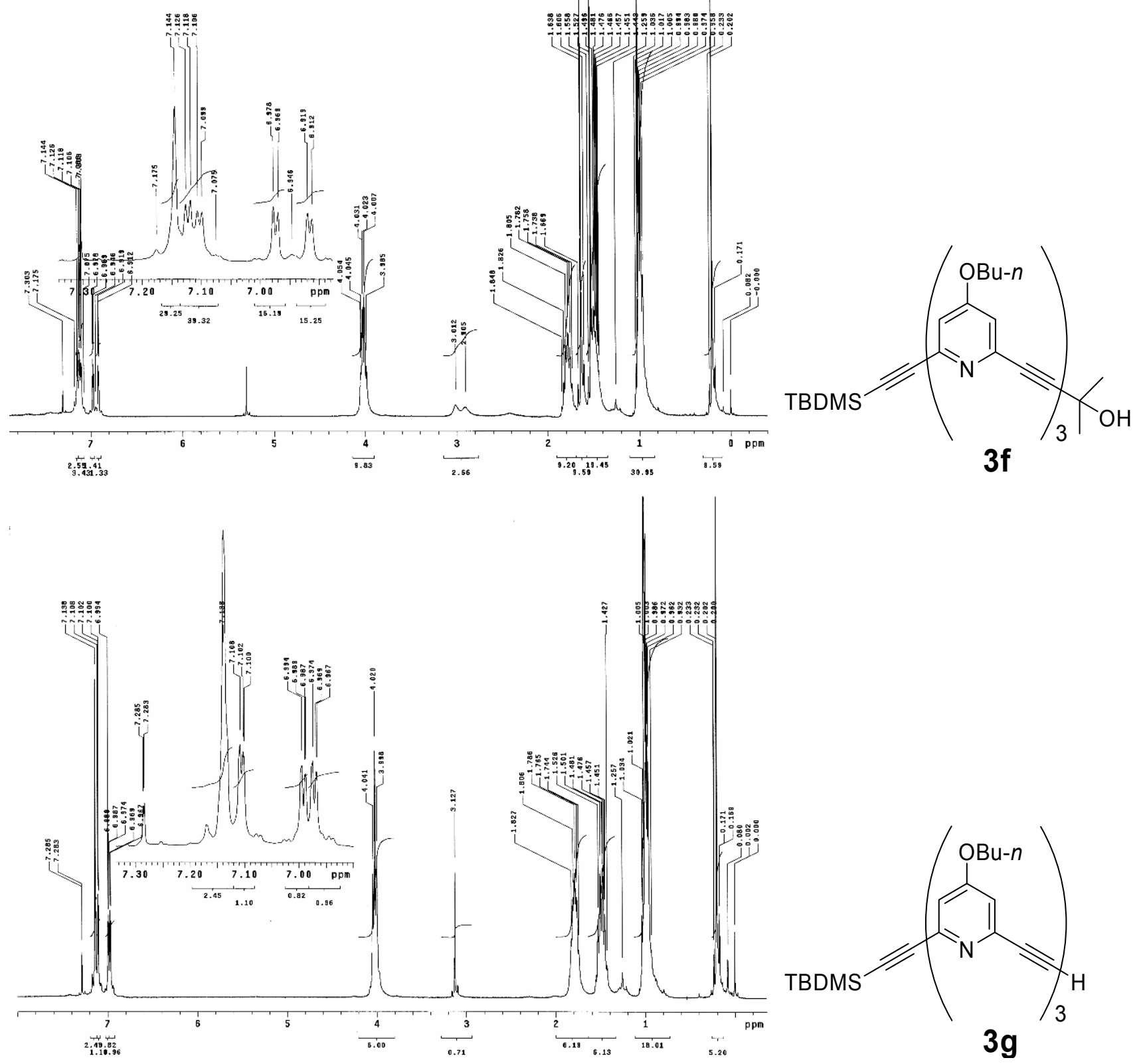

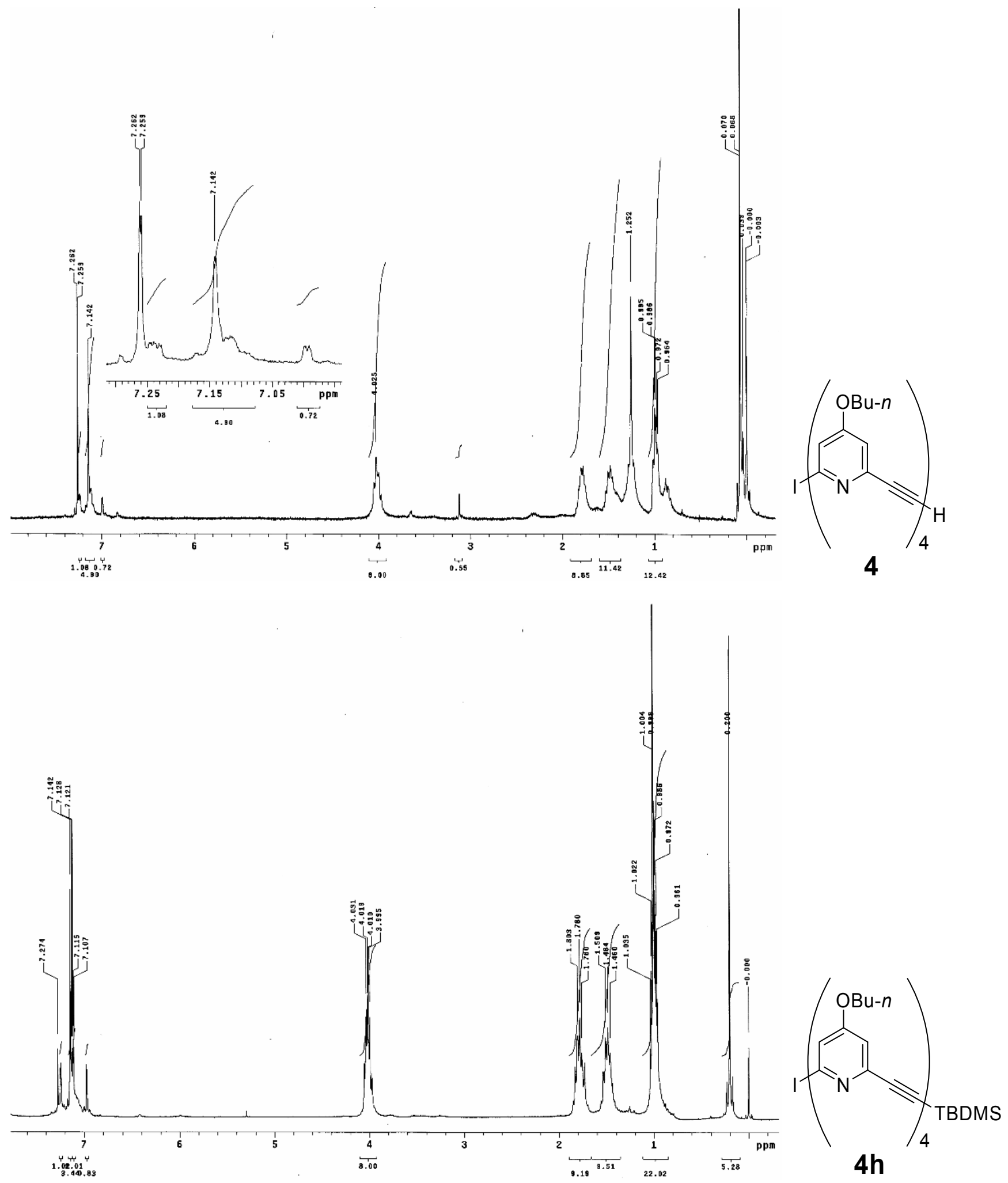


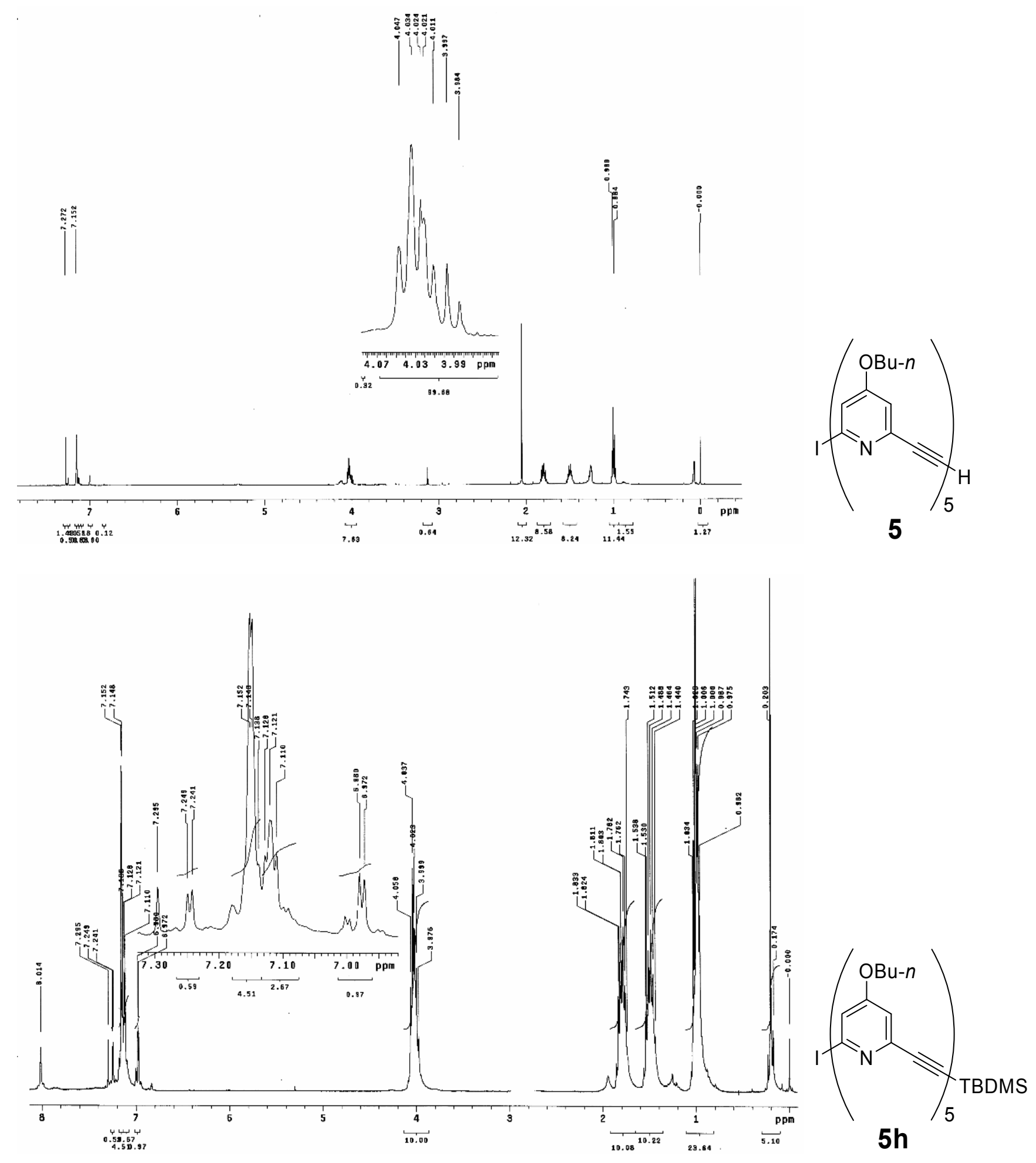



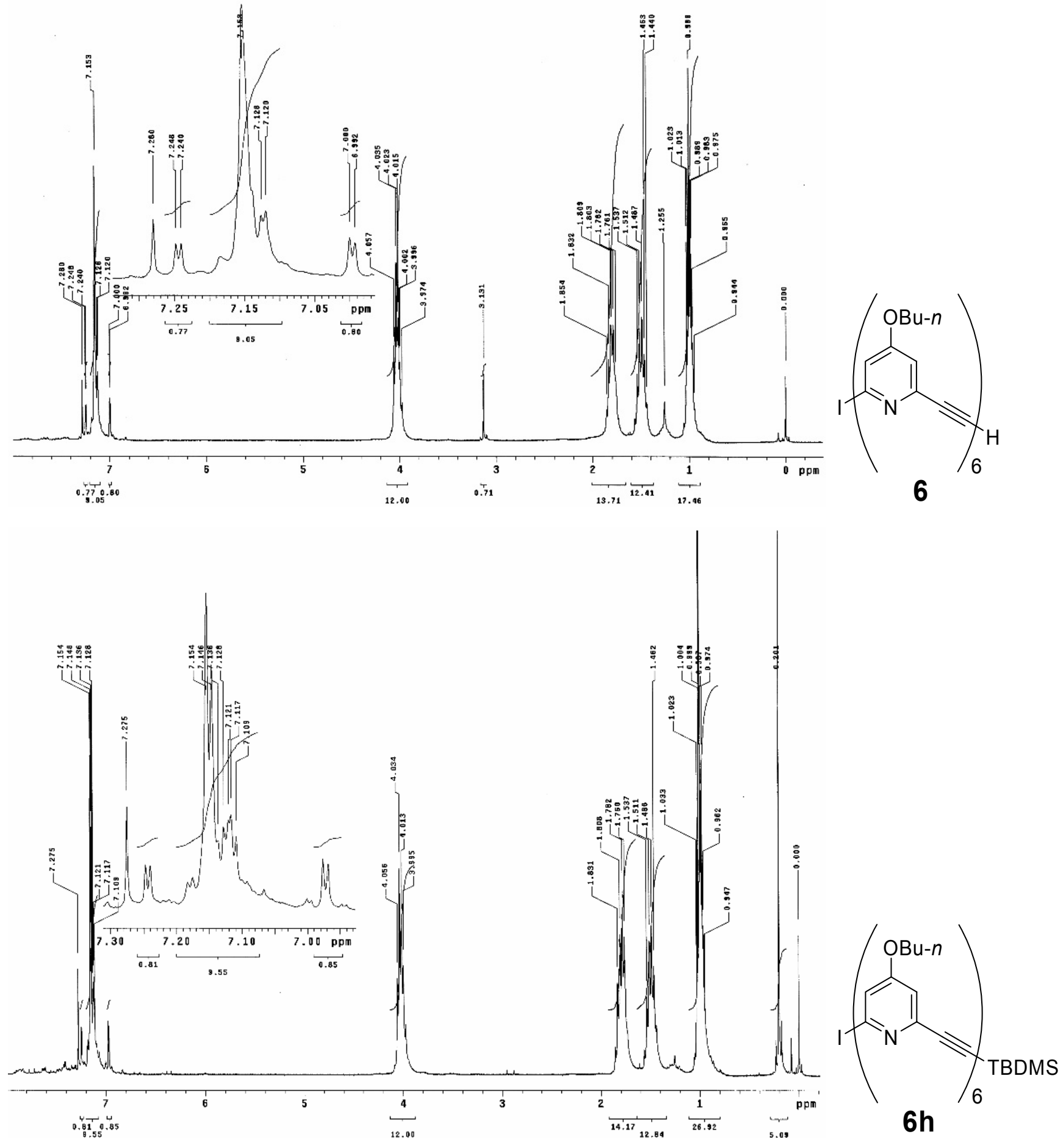

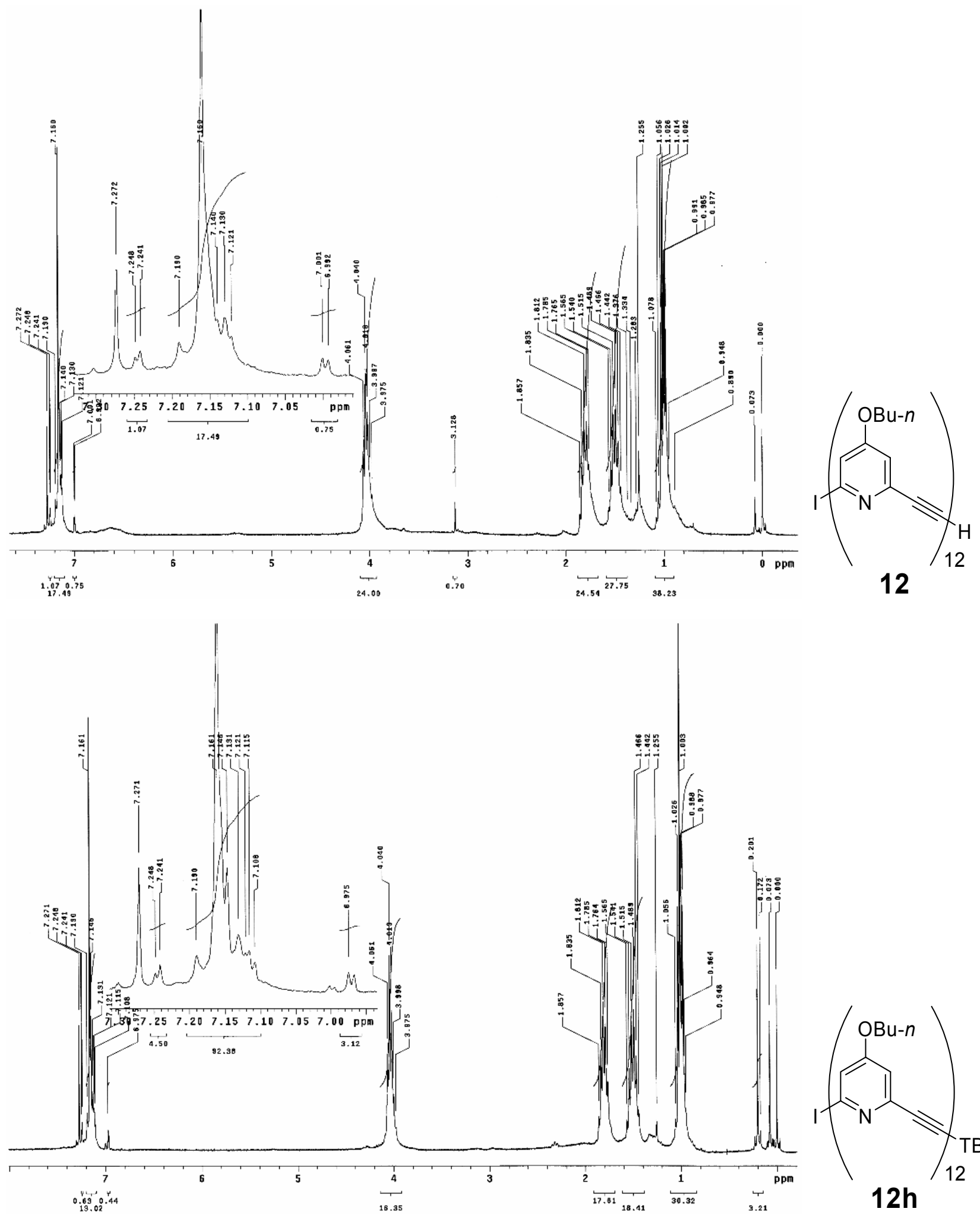

12

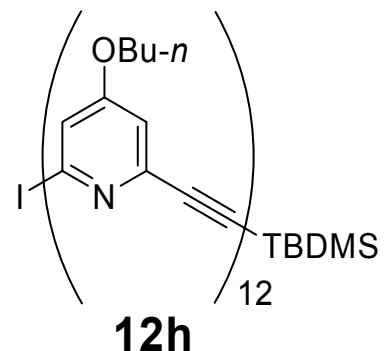



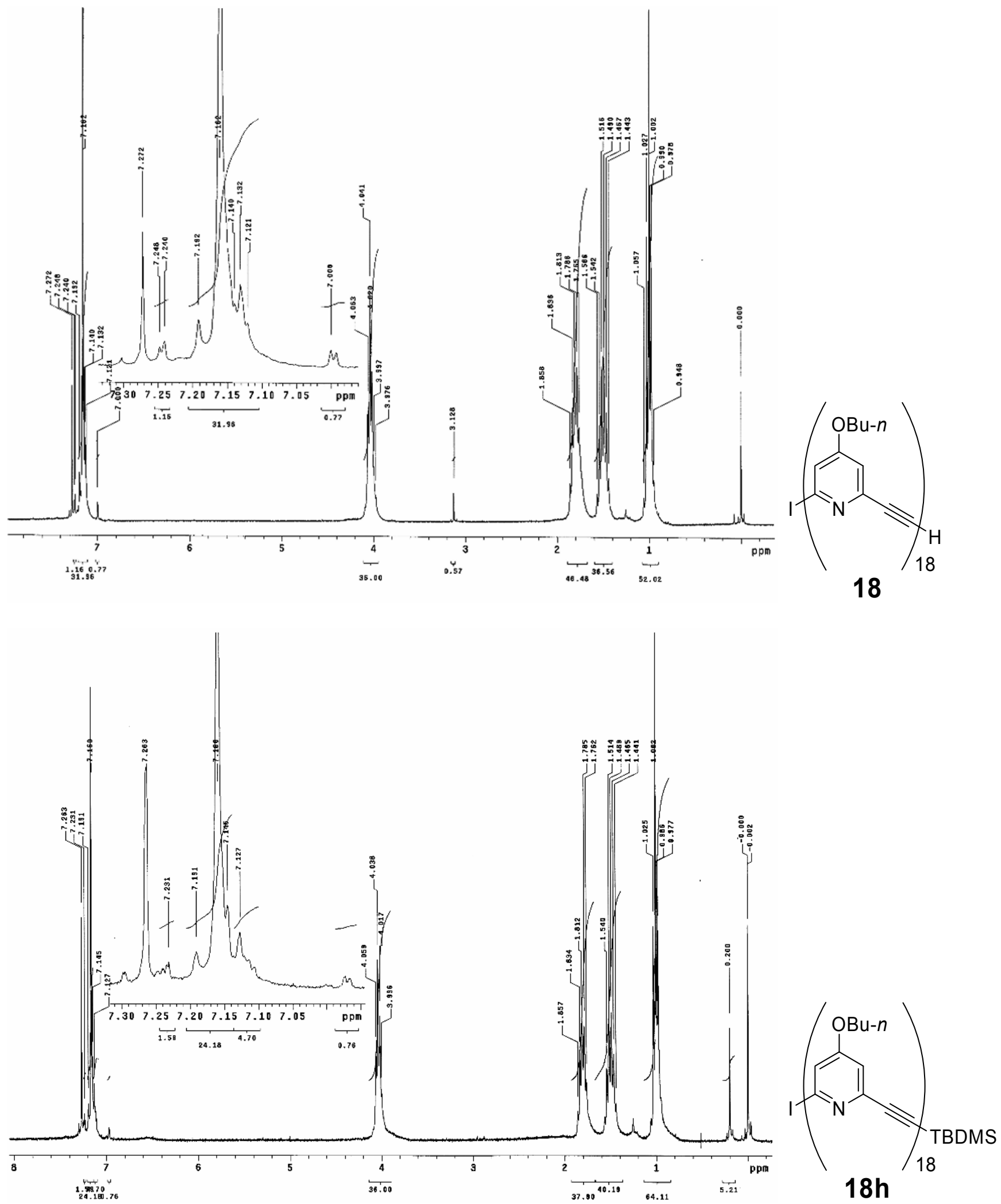

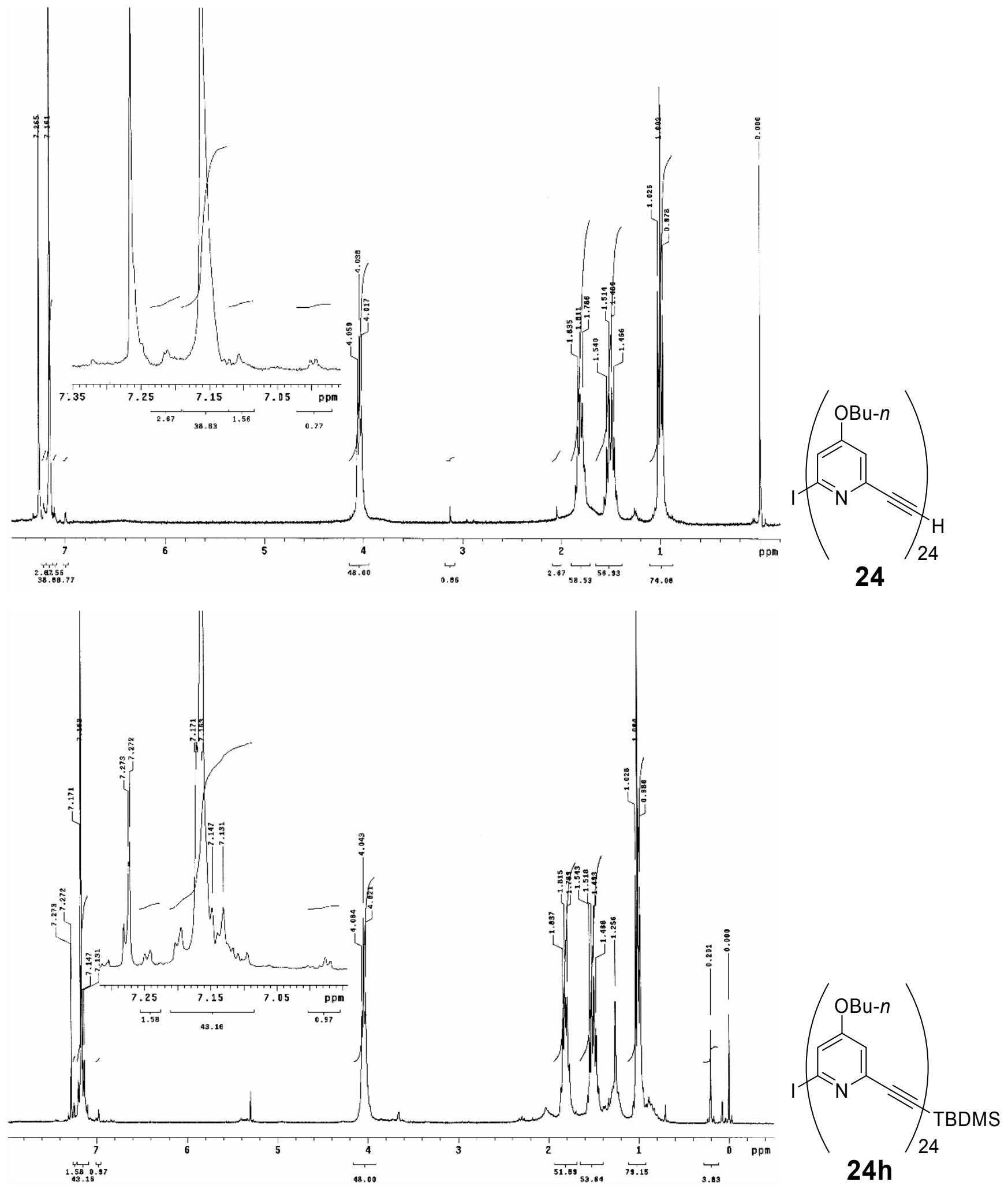